\title{
Cibo e rifugiati nella città capitolina, tra pratiche di emergenza e tentativi di agentività
}

Food and Refugees in the Capitoline city, between emergency and agency

\section{Donatella Schmidt e Giovanna Palutan}

\section{(2) OpenEdition \\ Journals}

\section{Edizione digitale}

URL: http://journals.openedition.org/aam/854

DOI: $10.4000 /$ aam.854

ISSN: 2038-3215

Editore

Dipartimento Culture e Società - Università di Palermo

\section{Notizia bibliografica digitale}

Donatella Schmidt e Giovanna Palutan, «Cibo e rifugiati nella città capitolina, tra pratiche di emergenza e tentativi di agentività », Archivio antropologico mediterraneo [Online], Anno XXI, n. 20 (2) | 2018, online dal 31 décembre 2018, consultato il 23 septembre 2019. URL : http:// journals.openedition.org/aam/854; DOI : 10.4000/aam.854

Questo documento è stato generato automaticamente il 23 septembre 2019.

Archivio antropologico mediterraneo 


\title{
Cibo e rifugiati nella città capitolina, tra pratiche di emergenza e tentativi di agentività
}

Food and Refugees in the Capitoline city, between emergency and agency

\author{
Donatella Schmidt e Giovanna Palutan
}

\section{Note introduttive}

1 Ci piace iniziare questo saggio con le parole di M.F. Fisher, giornalista del The New Yorker e autrice, in piena seconda guerra mondiale, del libro The Gastronomical Me perché la stessa domanda che, al tempo, avevano posto a lei potrebbe oggi essere posta a noi: perché in un momento di crisi scrivere sul cibo? Perché fra i tanti temi possibili scegliere proprio questo? Questo saggio intende affrontare il tema del cibo in relazione con il tema dei rifugiati in un momento in cui tanto la società italiana quanto più in generale la UE lo vede generatore - anche se sarebbe più corretto dire evidenziatore di una crisi profonda che disgiunge gli ideali dalle pratiche politiche, l'interesse del singolo stato vis-à-vis la visione corale. Si colloca dunque in una problematica più ampia che investe molte capitali europee: l'emergenza di persone che in fuga da conflitti e lotte intestine o da un senso d'insicurezza dalle matrici più diverse, sono arrivate nel nostro continente al fine di ottenere una qualche forma di protezione. Pur inserendosi in un quadro generale, l'attenzione del nostro lavoro si volgerà a un contesto molto specifico, vale a dire quello di pratiche legate al cibo in due esperienze d'accoglienza rivolte a rifugiati nella periferia di Roma. Il primo caso riguarda un'esperienza di ospitalità civica chiamata Baobab; il secondo riguarda una mensa, gestita dai Gesuiti, nota come Centro Astalli. Pur essendo distanti per modalità organizzative, riteniamo che entrambe le esperienze siano comparabili in quanto la loro attenzione non è circoscritta all'aspetto nutritivo, ma abbraccia la persona nella sua interezza adottando una visione in cui le necessità materiali non sono scindibili da quelle simboliche della commensalità. Da qui va rilevata la parte innovativa della 
nostra ricerca: mentre la letteratura etnografica nei due ambiti presi singolarmente è copiosa - ossia cibo da un lato e rifugiati dall'altro - l'unione dei due temi è a tutt'oggi scarsamente presente. Quando poi il binomio cibo e rifugiati viene affrontato, la prospettiva è in termini di sicurezza alimentare con l'accento rivolto più all'accesso al cibo che all'aspetto simbolico e relazionale che invece a noi interessa affrontare.

Il saggio qui presentato si divide in tre parti: la prima offre una descrizione etnografica ${ }^{1}$ dei due casi di studio considerati, preceduta da alcune precisazioni di metodo e da una sintesi sullo stato dell'arte nei due ambiti di studio, cibo e rifugiati; la seconda è rivolta a una loro comparazione; la terza mira a una riflessione che suggerisce una possibile chiave interpretativa delle modalità di interazione alla luce del concetto di agentività. In questo elaborato, la nostra attenzione sarà rivolta alle voci di attivisti e di volontari, riservando a un altro contributo il compito di dare ampio spazio all'altra parte, costituita da rifugiati e richiedenti asilo coinvolti nella ricerca.

\section{Metodo e stato dell'arte}

\subsection{Note sul metodo}

3 I dati etnografici presentati fanno parte di una ricerca tutt'ora in corso, condotta all'interno del progetto FOR - Food and Refugees - che si svolge in alcune città italiane ed europee tra cui Roma e Berlino. Il progetto non intende incentrarsi tanto sulle difficoltà, che sono una costante dell'interazione quotidiana fra volontari e rifugiati, quanto su modalità creative e propositive di affrontarle. L'accesso al campo nei due contesti scelti si è realizzato con modalità differenti. L'ingresso alla mensa del Centro Astalli è avvenuto attraverso vie istituzionali e si è strutturato in una fase preliminare di contatti con i responsabili dell'Astalli e della mensa per raccogliere informazioni sulla struttura e presentare il progetto. La ricerca è stata condotta con la richiesta, da parte dei responsabili, di una presenza di tipo covert in quanto la tipologia di utenti era caratterizzata da una particolare vulnerabilità. Si è quindi riflettuto su come muoversi in un contesto contraddistinto dallo spirito di "non-fare domande", in cui le interazioni fra rifugiati e volontari erano sì votate alla cordialità, ma circoscritte al momento della distribuzione del cibo. Il lavoro di Irene Glasser (1998), presso la Tabernacle Soup Kitchen for homeless peoples in New England, ha fornito utili spunti in quanto, come la nostra, la sua ricerca doveva essere condotta in modo tale da non disturbare la routine dei frequentatori della cucina popolare. Il ruolo della ricercatrice è dunque a metà strada fra l'essere coperta (rispetto all'utenza) e scoperta (rispetto ai volontari e agli operatori). Dopo un primo periodo di osservazione lavorando come volontaria durante la distribuzione dei pasti (due giorni al mese da agosto 2016, al bancone del self service e nei locali in cui si consuma il pranzo), una delle due ricercatrici, Giovanna Palutan, ha cominciato a riflettere sulle dinamiche osservate attraverso interviste, strutturate e informali, a operatori e a volontari della struttura, continuando in parallelo a prestare servizio durante la distribuzione dei pasti.

4 Per quanto riguarda il Baobab, le ricercatrici hanno cominciato nel 2015 con visite informali durante i momenti della distribuzione dei pasti per osservare le dinamiche in gioco; in un secondo momento, hanno presentato il progetto di ricerca ai referenti dell'associazione, chiedendo di essere presenti o di prestare servizio durante i pasti. La risposta degli attivisti è stata di tipo informale: le ricercatrici erano libere di venire 
quando desideravano e di interagire con i volontari e con gli ospiti. La presenza nel campo è stata dunque overt, ossia dichiarata. Nel presidio di via Cupa, Giovanna Palutan ha lavorato come volontaria alla distribuzione dei pasti in agosto e settembre 2016 mentre, a partire dal 2017, entrambe le ricercatrici si sono alternate in sopralluoghi mensili al presidio che, nel frattempo, era stato spostato dietro la stazione Tiburtina, in piazzale Maslax. Qui hanno cominciato a condurre interviste in profondità ai volontari dell'associazione, in particolare alle persone addette al cibo. Riguardo agli attivisti, che avevano seguito tutta la vicenda del Baobab e che avevano quindi una memoria storica delle varie fasi - centro Baobab, via Cupa, piazzale Maslax - le interviste sono state condotte attraverso la tecnica del photoeliciting (Epstein et al. 2006), ossia la narrazione degli interlocutori si è sviluppata commentando una serie di immagini scelte da servizi giornalistici di testate nazionali (la Repubblica o l'Espresso) o scaricate dalla pagina facebook dell'associazione che documentavano le attività nel corso di più anni, dal 2015 al 2018. Questa tecnica di ricerca è stata scelta per la sua potenzialità nell'esplorare $\mathrm{i}$ significati attribuiti a luoghi, situazioni, persone, oggetti e materiali - incluso il cibo rappresentati nelle immagini e per il ruolo attivo degli interlocutori nel corso dell'intervista. Una parte delle interviste ai volontari e agli attivisti è stata condotta nel campo, sotto lo sguardo degli ospiti, che a volte si avvicinavano alla ricercatrice per dialogare con lei. Va considerato che, durante tutto il 2015, i migranti erano in transito (centro Baobab), pertanto c'erano difficoltà di tipo logistico e linguistico ad avere una comunicazione non mediata in quanto la maggioranza parlava solo in tigrino; nel 2016 (presidio di via Cupa con Baobab Experience) i rifugiati erano in movimento nel tentativo di attraversare le frontiere. Nel 2017, dopo l'ennesimo sgombero, il presidio di emergenza veniva spostato, come già detto, in un'area dietro la stazione Tiburtina (piazzale Maslax) diventando un'esperienza più stanziale e con una presenza di persone di provenienza diversa oltre il Corno d'Africa e il Sud Sudan. Tale precaria stanzialità ha permesso un più facile avvicinamento di alcuni ospiti, che a loro volta erano divenuti volontari.

\subsection{Stato dell'arte}

5 La bibliografia riguardante l'aspetto sociale e simbolico del cibo in ambito antropologico è, come è noto, ricca e articolata e fra i classici ci limitiamo a ricordare $i$ lavori di Lévi-Strauss, di Mary Douglas e di Marvin Harris. In tempi più recenti e con un'ottica che si avvicina alle finalità della nostra ricerca, ricordiamo Mintz con il suo focus su status e potere correlati al cibo (Mintz 1996); Carole Counihan che ha condotto il suo lavoro seminale su cibo e cultura (Counihan, Van Esterik 2013); Diana Mata Codesal che ha analizzato pratiche legate al cibo come strategia sensoriale per combattere il senso di frammentazione causato dalla migrazione (Mata Codesal 2008); Cramer, Greene e Walters che si sono interessati alle modalità comunicative espresse attraverso il cibo (Cramer, Greene, Walters 2011); Lidia Marte che ha esplorato il concetto di produzione di una visibilità storica attraverso forme di autonomia connesse ai condimenti (Marte 2008). Il cibo in molte occasioni ha rappresentato la base dell'azione politica e sociale di movimenti ad esso legati: Counihan e Siniscalchi parlano di food activism che coniuga l'iniziativa spontanea con quella istituzionale e l'attivismo individuale con quello collettivo (Counihan, Siniscalchi 2014); Orlando guarda ai movimenti sociali legati al cibo in termini di solidarietà (Orlando 2015). 
6 Per quanto riguarda il tema di rifugiati e richiedenti asilo in ambito socioantropologico, a livello internazionale si possono individuare alcune linee direttrici: 1) gli studi seminali di Harrell-Bond sui limiti del cosiddetto humanitarianism (HarrellBond, Voutira 1992, Harrell-Bond 2005), le ricerche di Liisa Malkki sulle forme di intervento umanitario (Malkki 1996 con lo scenario di Rwanda e Burundi e Malkki 1995a con lo scenario dei rifugiati in Tanzania) e la disanima critica sulla ragione umanitaria di Fassin e di Mariella Pandolfi (Fassin e Pandolfi 2010; Fassin 2012); 2) le difficoltà dell'Europa nel rispondere alle continue "crisi di rifugiati" (Green 2013; Facchini 2018) espresse da un respingimento di persone in aree a rischio attraverso accordi con regimi repressivi che minano la tradizione umanitaria europea (Webber 2017; Schiocchet 2018); 3) una ricerc-azione su forme di advocacy in favore di rifugiati e richiedenti asilo (Taran et al. 2016) e movimenti di visibilità messi in atto da parte di rifugiati (Napuli 2015 e Borri 2017 su Berlino); 4) ricerche su accampamenti informali (Agier 2018 sulla Jungle a Calais; Queirolo Palmas 2017 sulla bulle a Parigi; Martinelli a Roma, 2008), su campi di rifugiati (Terzioglu 2018; Melissa Wall et al. 2017) e sugli hotspots (Dimitriadi 2017 per la Grecia; Sciurba 2017 sulla Sicilia); 5) la criminalizzazione della solidarietà da un lato (Liz Fekete 2018) e la risposta delle Chiese e di parte della società civile dall'altro (Giordan, Zrinščak 2018; Schmidt, Palutan 2018). Infine, vanno rilevate proposte costruttive come quelle di Stefano Allievi relative alla necessità di un ritorno alla ragionevolezza nella gestione della questione migratoria riaprendo canali regolari e sottraendo il settore alle mafie transnazionali (Allievi 2018) e riflessioni impietose come quelle di Zygmunt Bauman che assegna agli stranieri venuti da lontano il compito di ricordarci in modo esasperante quanto sia fragile il nostro benessere (Bauman 2016) ${ }^{2}$.

7 In Italia, il dibattito antropologico sul tema dei rifugiati è stato introdotto per la prima volta da due numeri monografici dell'Annuario di Antropologia, il primo curato da Mauro Van Aken (2005), l'altro curato da Barbara Pinelli (2013a). Da allora il panorama si è andato arricchendo e ha acquisito sempre più una fisionomia specifica all'interno del più ampio spettro delle ricerche sulle migrazioni. Una revisione della letteratura socioantropologica prodotta negli ultimi anni delinea alcune principali aree di interesse tematico: 1) la ricostruzione degli scenari politici da cui provengono i rifugiati (Ciabarri 2016; Costantini, Massa e Yazdani 2016; Manocchi e Marchetti 2016; Jourdan 2012; Campesi 2011); 2) la costruzione della figura del rifugiato da parte delle strutture burocratiche e di accoglienza (Van Aken 2005; Vacchiano 2005); 3) la dimensione giuridica e dei diritti (Gozzi, Sorgoni 2010; Morone 2011; Paternò 2014) e di genere (Pinelli 2013b); 4) il sistema di accoglienza per richiedenti asilo (Sorgoni et al. 2011; Meloni 2012; Marchetti 2016); 5) la riflessione sulle esperienze empiriche da parte di ricercatori-operatori coinvolti a vario titolo nelle strutture ordinarie e straordinarie d'accoglienza (Campesi 2015; Gallotti, Tarabusi 2018; Biffi 2018); 6) gli studi sui centri di identificazione e di espulsione (Ravenda 2011); 7) la narrazione (Costantini 2012) e la lettura soggettiva dei rifugiati (Sbriccoli, Perugini 2012; Sbriccoli, Jacoviello 2012; Federici, Degli Esposti Merli 2014); 8) il tema delle occupazioni (Meloni 2012) e degli insediamenti spontanei (Queirolo Palmas 2017; Manocchi, 2012; Oteri, Carrera 2012) e, più in generale, dei campi (Rahola 2003; Pinelli 2017); 9) il tema degli sbarchi (Pinelli, Ciabarri 2017) e degli arrivi in Italia dal punto di vista dell'impatto mediatico (Cuttitta 2012, Gatta 2012). Infine, vanno segnalati spunti riflessivi che richiamano la responsabilità delle discipline antropologiche (per esempio Palumbo 2011; Altin, Sanò 
2017; Fontanari 2017; Riccio, Tarabusi 2018) nell'offrire uno sguardo attento e un contributo solido a questo importante tema della contemporaneità.

Nonostante la letteratura in questi due ambiti - rifugiati e richiedenti asilo da un lato e cibo dall'altro - sia qualitativamente e quantitativamente corposa, l'unione dei due temi che costituisce la nostra linea di ricerca, affronta uno specifico settore ancora poco esplorato. Quando poi viene preso in considerazione, il binomio viene studiato nei termini della cosiddetta food security, in particolare nei campi profughi; l'accento viene cioè posto sull'accesso al cibo (vedi a questo proposito i due interessanti lavori di Kiptinness, Dharod 2011 e di Kinyua 2013) e non sull'aspetto simbolico e relazionale ad esso legato. Alcune poche eccezioni sono costituite dai saggi pionieristici di Hannah Lewis che si è occupata di cibo, di eventi e di comunità immaginate fra rifugiati accolti in Inghilterra (Lewis 2010) e da ricerche sugli orti comunitari come mezzi di ricostruzione di appartenenze nei luoghi di migrazione (Harris, Minniss, Somerset 2014).

\section{Baobab. Un modello di solidarietà dal basso}

\subsection{Centro Baobab}

Era la cucina il centro nevralgico e affettivo del Baobab.

Roma. Via Cupa è una traversa lunga e stretta situata in un'area manifatturiera abbandonata tra i rioni Bologna e San Lorenzo che termina di fronte allo storico Cimitero del Verano, nella transitata via Tiburtina. Qui, nei primi anni del 2000, veniva aperto un centro d'accoglienza per rifugiati e richiedenti asilo con annesso un centro culturale e un ristorante eritreo. Il Centro Baobab, così si chiamava, era un'associazione gestita da un gruppo di rifugiati che lavorava in convenzione con il Comune, conosciuta per la sua cucina e per essere un punto di riferimento in particolare per le collettività del Corno d'Africa ${ }^{3}$.

Quando, nel 2015, avviene il grande esodo di eritrei ed etiopi (si veda Gebrewold e Bloom 2016) che tentano di raggiungere i paesi del nord d'Europa - la Svezia in particolare - il Centro Baobab diviene parte integrante di quella particolare mappa di viaggio, costruita su esperienza e passaparola, di chi ha deciso di intraprendere il lungo cammino. La sua posizione è strategica: vicina allo snodo ferroviario della Tiburtina, alla stazione delle corriere, a pochi metri dalla metropolitana e a diversi percorsi autobus, ma al tempo stesso in una via secondaria, marginale, nascosta alla vista. Così, in quell'estate al Baobab passano in 35.000. Sono principalmente giovani uomini, ma anche minori soli, giovani donne, a volte incinte. Li chiamano i transitanti perché sostano, si rifocillano, ripartono. Per le autorità cittadine sono "gli invisibili": nessun interesse a trattenerli, nessun obbligo a offrire un servizio. Ma tre pasti al giorno con una media di tre giorni ciascuno fanno quasi 350.000 pasti, un'enormità. Incapaci di far fronte a una simile emergenza da soli, i gestori del Baobab chiedono aiuto alla cittadinanza che risponde. Viola, una delle pioniere del Baobab, descrive l'esperienza nei seguenti termini:

Una grande rete di solidarietà: [Al Baobab] era un continuo scaricare cibo, scatolame. C'erano i banchetti del mercato di Zagarolo che ci portavano l'invenduto, c'erano i ristoranti, i catering, i forni di pane e pizza. C'erano anche le diecimila enjer $a^{4}$ che ci mandavano i ristoranti eritrei. [...] Il magazzino era una cosa mai vista. Enorme, con quattro pareti alte, stracolme di roba. Uno entrava e diceva: 
madonna quanta roba! Ma da un giorno all'altro spariva. I numeri erano così alti: hai voglia a pasta, hai voglia a tonno (Viola, intervista con photoeliciting, 2 novembre 2017). Vaticano. Non si risponde solo con cibo, con vestiti e con beni di prima necessità, ma semplici cittadini si affacciano al Baobab e si offrono come volontari:

Sono arrivata lì a metà giugno: la cosa era cominciata da una settimana, forse due. Avevo letto la notizia su facebook, ero andata con un sacchetto di mele e poi non me ne sono più andata (Viola, ibidem).

I volontari, velocemente e alacremente, mettono in piedi una gestione dell'emergenza: i duecento posti del centro di accoglienza vengono destinati alle donne e alle famiglie, agli uomini è messo a disposizione il cortile che di sera diventa un dormitorio. I locali del Baobab vengono riorganizzati, assegnando a ciascuno nuove funzioni: il magazzino per lo stoccaggio del cibo, la stanza riservata a vestiti donati, l'infermeria, l'area gioco per i bambini. Una parte del ristorante viene dedicata agli arrivi: qui viene consegnato un kit con il necessario per l'igiene personale e un foglio con le informazioni sul Baobab tradotte in inglese e in tigrino:

Spiegavamo che eravamo volontari perché questa è una cosa che all'inizio non capivano. A volte si creavano dei momenti di tensione in cui pretendevano delle cose. Ma io dicevo: "noi siamo volontari, non ci paga nessuno. Quello che non c'è mi dispiace, ma non c'è. Quello che c'è, ringraziamo che c'è. [...] spiegavamo anche che non tolleravamo la violenza, nessuna discriminazione, nessuna prevaricazione dell'uomo sulla donna, cosa che a volta capitava anche sulle volontarie (Viola, ibidem).

\section{quotidianità}

Ci tenevamo che loro aiutassero nella preparazione dei pasti, nelle pulizie e nella gestione, che si facesse sempre assieme e che non ci fosse assistenzialismo (Viola, ibidem).

14 Nei locali ogni giorno è un continuo via vai di persone: migranti in arrivo e in partenza, volontari impegnati nelle varie attività, ospiti che prestano servizio, cittadini che portano donazioni in cibo e vestiti, medici, avvocati di strada. In quella rete imponente di solidarietà dal basso che si andava intessendo e che gestiva un'emergenza così sempre di corsa, trattenendo il fiato, avvengono anche incontri inaspettati:

C'erano scene commoventi: chi si era perso nei naufragi e si ritrovava lì o, arrivando, rivedeva i vicini di casa che erano partiti prima (Viola, ibidem).

È la cucina il luogo nevralgico del Centro Baobab, è lì quello che Sonia - volontaria dai primi giorni dell'emergenza - chiama «il centro affettivo»:

La preparazione del pasto era il momento clou. Avevamo una media di 700 persone, con picchi sugli 850, e preparavamo tre pasti al giorno: colazione, pranzo, cena. Era una cosa impegnativa, che prendeva tanto tempo, però era anche uno dei momenti più belli [...] uno dei compiti più ambiti da tutti i volontari. La cucina era molto grande, c'erano due cuoche e, a turno, una ventina di volontari e di ospiti che aiutavano. Era un momento di condivisione: mentre affetti e fai le cose, chiacchieri e fai amicizia. [...] Cercavamo sempre di dare cibo di una certa qualità. Certo, con quei numeri non era semplice, però il cibo era importante per dare dignità. [...] Quando riuscivamo a farci dare l'injera le due cuoche Maeron e Selam facevano lo zighini, il piatto principale eritreo ed etiope: una specie di spezzatino, speziato e piccante, con sugo, ceci e patate. Poi con altra enjera si mangiava tutto (Viola, ibidem). 
Le due cuoche, che prima cucinavano nel ristorante del centro culturale, ora cucinano per i migranti, rispettando le preferenze e i divieti alimentari - niente carne di maiale o vino nel cibo - e, la sera nel periodo del Ramadan, a chi osservava il digiuno si dava un sacchetto con del cibo per il pasto della notte. Questa esperienza di solidarietà era definita dai media come un modello di accoglienza pacifica costruito dal basso. Nonostante le difficoltà, una bella storia ${ }^{5}$.

\subsection{Baobab Experience, via Cupa}

Da essere il momento più bello la preparazione del cibo era diventato il momento più faticoso.

A dicembre 2015 il Centro Baobab viene chiuso dalla municipalità ufficialmente per questioni igieniche. Tuttavia, con l'inizio della primavera i migranti - forse perché il Centro era ancora segnato sulla loro mappa di viaggio, forse perché non sapevano dove andare - iniziano nuovamente ad arrivare. Così un gruppo di volontari decide di raccogliere l'esperienza di quei mesi e di fondare l'associazione Baobab Experience. E il Baobab riprende vita, ma non più dentro una struttura bensì sulla strada, dove si montano delle tende o si stendono materassi:

La sera mettevamo delle sedie a chiudere la strada da una parte e dall'altra. Alle sette si smontava tutto, svegliavamo i ragazzi prima che aprissero i negozi e la strada veniva riaperta. C'era questo accordo tacito con i vicini (Viola, ibidem).

Dal momento che la cucina era chiusa e inagibile, i volontari prendono una decisione difficile: si sarebbe preparato il cibo a casa e portato lì in via Cupa, organizzandosi. $\mathrm{E}$ poi certo si sarebbe continuato a bussare alla porta dei forni di pane, chiedendo l'invenduto, alla porta di mense e di ristoranti chiedendo l'avanzato.

C'era il problema dei pasti: farli a casa e portarli qui. [...] tu fai due chili, tu fai tre chili, tu porti le uova [...] Poi quando il cibo non bastava, si faceva l'insalatona con del cibo in scatola che si teneva nel mobiletto [...] però da essere il momento più bello preparare il cibo era diventato il momento più faticoso (Viola, ibidem).

Era stressante [...] c'era sempre questa ansia di non sapere se c'era cibo per tutti (Sonia, volontaria, Boabab Experience, intervista 17 gennaio 2018).

Nonostante le difficoltà ${ }^{6}$, i pasti vengono distribuiti:

All'una si inizia con il pranzo. La fila si forma in un baleno e si srotola per una decina di metri lungo la strada stretta. Su un tavolo ci sono colonne di piatti di plastica e il cibo preparato dai volontari: un contenitore pieno fino all'orlo di pasta fredda condita con verdure sottaceto, olive e mais. Due volontari riempiono i piatti di pasta e, passandoceli, ci chiedono di aggiungere il pane. A nostra volta li passiamo a due ospiti del campo che distribuiscono i piatti di cibo alle persone in fila. Accanto al tavolo, l'ospite più anziano bada che non ci siano incidenti e coordina il lavoro parlando in tigrino. Le persone si siedono a mangiare lungo il muro della strada, ciascuno per conto proprio. È un pasto affrettato, silenzioso (Palutan, note di campo, primo settembre 2016).

20 Le persone che nell'estate del 2016 sostano in via Cupa provengono principalmente dall'Eritrea e dal Sud Sudan. In prevalenza si tratta di uomini, ma ci sono anche giovani donne - che nella quasi totalità hanno vissuto esperienze traumatiche durante il viaggio - minori soli e famiglie oromo, un gruppo perseguitato in Etiopia. Sono arrivati in Italia attraversando il Mediterraneo centrale, anche loro con l'intenzione di proseguire verso altri paesi europei dove li attendono i familiari. Il presidio di via Cupa per loro dovrebbe essere un luogo di passaggio dove riposare per pochi giorni e per 
pianificare l'ulteriore tragitto da compiere. Tuttavia, a differenza dei transitanti dell'anno prima, sono stati quasi tutti fotosegnalati negli hotspots di frontiera perché nel frattempo lo scenario europeo è cambiato: vari paesi avevano introdotto controlli all'interno dell'area Schengen. Come conseguenza le frontiere settentrionali dell'Italia erano chiuse e non era più possibile il passaggio verso Francia e da lì verso Germania o Svezia. Chi è dovuto tornare indietro è carico di frustrazione e non solo perché la vita per strada è piena di disagi, ma perché il viaggio forzatamente interrotto causava una sensazione di instabilità sia fisica che mentale paralizzante ${ }^{7}$. Sale la tensione, le istituzioni sono latitanti, scoppiano anche le risse e la polizia comincia a far sentire la sua presenza:

Quando eravamo in strada hanno cominciato a fare le identificazioni a manetta.

Una settimana sì e una settimana no. [...] Bloccavano gli ingressi della strada, se li portavano tutti via e poi li rilasciavano (Andrea Costa, attivista di Baobab Experience, intervista 11 febbraio 2018).

21 Da transitanti a tempo determinato gli ospiti di via Cupa vengono trasformati in stanziali a tempo dilatato. Per far fronte a questo tempo dilatato, che andava riempito, $\mathrm{i}$ volontari del Baobab improvvisano corsi d'italiano, organizzano partite di calcio, propongono visite guidate nella capitale, accompagnano al mare. Il modello d'accoglienza, che ormai aveva preso forma, era esemplificato dallo slogan «welcome with dignity».

\subsection{Baobab Experience, piazzale Maslax}

Il cibo potrà essere più o meno gradito, ma anonimo mai.

In ottobre 2016 il Comune di Roma smantella via Cupa e la maggioranza dei migranti viene dispersa nei parcheggi abbandonati dell'area circostante. Non appena piccoli assembramenti di persone si raccolgono attorno a dei volontari che distribuiscono cibo e vestiti, arriva la polizia a disperderli o a chiedere i documenti. Così una dispersione dopo l'altra questo gioco pesante di "guardie e ladri" lascia tutti - volontari e migranti - esausti. Pian piano il gruppetto di migranti si sposta in un parcheggio dietro la stazione Tiburtina e da lì, sempre pian piano, si accampa in un luogo appartato poco lontano detto piazzale Maslax.

La luce del tardo pomeriggio invernale sta cedendo il posto alla penombra. Le tende appaiono ancora più appiattite sull'asfalto di quello che era un grande parcheggio. Sono molte - ne conto oltre un'ottantina - c'è vita attorno ad alcune: attorno a dei fuochi accesi qua e là siedono gruppetti di uomini a scaldarsi, a conversare. Fa già freddo. A sinistra il lungo tendone bianco - una donazione recente - funge da mensa e da ritrovo. È al buio. Poi, all'avvicinarsi dell'ora di cena, le luci vengono accese e si riempie di giovani uomini e alcune poche donne fino a divenire affollato. Ci sono sei tavoloni, tre da un lato e tre dall'altro, e un altro tavolo accanto all'entrata disposto frontalmente. In fondo, un gruppetto è accanto alle prese per la ricarica dei cellulari. Arrivano alcuni volontari con qualche piatto e poi il pentolone di minestra, i contenitori di pasta, il sacco di pane, le banane. Si forma velocemente la fila dei ragazzi, un'ottantina, forse un centinaio; il piatto di minestra passa nelle mani di un paio di volontari che aggiungono il pane e poi in quelle di un ragazzo che vi poggia una banana. Infine arriva nelle mani del giovane in attesa. Se qualcuno tenta di infilarsi, saltando la fila, è gentilmente inviato al fondo da una ragazza giovane, Myriam, molto attenta che tutto si svolga in modo ordinato. Sedute in disparte mangiano tre donne eritree, due giovani e un'anziana. Sono oggetto di attenzioni, ma solo da parte dei connazionali perché la comunicazione è esclusivamente in tigrino. Appena finito di mangiare, molti dei ragazzi si rimettono 
in fila, questa volta li aspetta un piatto di pasta. Pochi si siedono, la maggioranza consuma in piedi, in fretta (Schmidt, note di campo 21 febbraio 2018).

23 Anche in questa terza fase sperimentata dai volontari del Baobab il cibo continua a essere al centro dell'attenzione e della relazione. Procurato attraverso un'articolata rete di contatti, cucinato nelle varie case dei volontari con una distribuzione dei compiti affidata a facebook, caricato in auto, viene infine portato a piedi e a braccia negli ultimi duecento metri che separano gli spartitraffico di cemento dall'accampamento ${ }^{8}$. Potrà essere più o meno gradito, ma anonimo mai: ogni cuoca ha un nome, un volto, un suo modo di condire anche se a volte non è presente alla distribuzione che è affidata ad altri. E certo

Alcuni ragazzi hanno difficoltà a credere che nessuno di noi sia stipendiato. [...] Alla lunga, con la relazione, diventa evidente. La riconoscenza, l'affetto, quello che rimane loro lo toccano con mano. C'è la percezione di essere trattati in modo diverso (Sonia, intervista 17 gennaio 2018).

Dietro ogni piatto che arriva - a pranzo, a cena - ci sono una serie di volontari che l'hanno reso possibile, ciascuno facendo la sua parte, venendo incontro a persone che si trovano in un momento di precarietà prolungata:

Quello che offriamo noi è una tenda, un pranzo, la cena e amicizia, ma a livello di benessere, di bagni, di una doccia, di un letto, di una camera al coperto non c'è (Sonia, ibidem).

Com'è stata la relazione tra i volontari e i ragazzi nelle tre situazioni: centro Baobab, via Cupa e adesso al presidio di piazzale Maslax?

La situazione al centro Baobab era proprio diversa, con una grossa cucina, delle stanze e accanto, in un altro stabile, un dormitorio. Siamo partiti con 300 persone e siamo arrivati fino a 800: loro avevano la loro vita, il loro quotidiano nel dormitorio, mentre durante il pasto interagivano con noi. L'anno dopo, nel 2016, per strada in via Cupa è nato il desiderio di organizzare delle escursioni per la città, la gita a Villa d'Este ed è chiaro che la relazione c'è stata. Quindi il rapporto è diventato molto più personale ed è cambiata molto la composizione dei ragazzi che si appoggiavano a noi. [...] Quando interagisci gentilmente, chiedendo, le persone rispondono [...] ci stavano moltissimi livelli di risposta: semplice conoscenza, amicizia, innamoramento. Adesso, al presidio, non dico che tutti conosciamo tutti però una grande percentuale di ragazzi è identificabile ed è chiamata per nome (Sonia, ibidem).

26 All'alba del 13 novembre 2018 le forze di polizia hanno sgomberato anche l'accampamento di piazzale Maslax che nel frattempo era arrivato a contenere fino a 300 persone. Si è trattato del ventiduesimo sgombero nei tre anni di attività di Baobab Experience.

\section{Centro Astalli. Un modello di accoglienza strutturato}

Il Centro Astalli è un mondo articolato [...] La mensa [...] è considerata il cuore di questo mondo perché si è partiti da lì.

Nei locali della cucina è in corso la preparazione del pranzo che verrà servito alle 15.00. Pierpaolo, cuoco e responsabile della mensa, ci accoglie, dà indicazioni ai suoi aiutanti su quello che va fatto mentre è con noi e, dopo pochi minuti di attesa, ci porta nei locali dell'ambulatorio medico che in quel momento non è attivo. Ci fa sedere attorno a un grande tavolo: noi da una parte e Pierpaolo, divisa rossa e cappello bianco a falde, 
dall'altra. Dopo qualche battuta, gli chiediamo di raccontarci come organizza la cucina della mensa.

Questa mensa nasce come una cucina di emergenza - fornire un pasto caldo a un tot di persone che non sai mai quante sono. È una cucina italiana: si basa fondamentalmente sulla pasta. Ogni tanto gli faccio il cous cous ma non più di così. [...] Riso se ne fa, però gli afgani, gli iraniani usano qualità di riso diverse. [...] Hanno cotture diverse da noi. Morale della favola: spesso gradiscono di più gli spaghetti che il riso, nonostante poi si lamentino che in Italia c'è sempre pasta [...]. Abbiamo cercato di seguire delle circolari del Comune che davano indicazioni nutrizionali: un primo di pasta; un secondo generalmente di carne, ovviamente non carne di maiale: la maggior parte dei fruitori è di religione musulmana e nemmeno usiamo l'alcool nella cottura. Poi c'è un contorno: insalata, legumi. Una volta a settimana cerco di dargli pesce [...] bastoncini. Quello lo mangiano, altre cose no. Ho provato con le cozze, con i moscardini e mi venivano a dire: ma cosa mi hai dato? [...] Se dovessi fare un menu che piace a loro, dovrei fare esclusivamente pollo. Potrei farlo tutti i giorni, per dieci anni di seguito e non avrei problemi. Ho provato in passato, quando avevo più energia e li amavo di più, a fare delle cose più elaborate come timballi, sformati, ma poi c'è la diffidenza e ti chiedono: che ci hai messo dentro? C'è la carne di maiale? Ho imparato a fare cose che loro riconoscono. [...] In cucina mi aiuta un ragazzo curdo-iracheno qui dal 2000. [...] Poi c’è il mondo del volontariato: ci sono i novizi gesuiti e nel pomeriggio ci sono sette persone, a volte anche dieci. Dopo la venuta di papa Francesco c'è stata una grossa risonanza e le richieste di volontariato sono aumentate. ${ }^{9}$

L'intervista con Pierpaolo è avvenuta in una fase preliminare, in un momento di raccolta di informazioni sulla funzione della struttura e sulla sua storia mentre la ricerca vera e propria è cominciata un anno più tardi ${ }^{10}$. Le modalità della presenza della ricercatrice alla mensa sono l'esito di un lungo dialogo con i responsabili della struttura, la cui preoccupazione era che la presenza di un'osservatrice potesse creare disagio fra gli ospiti.

Pierpaolo ha condito la pasta con una salsa di verdure a base di cavolfiore, verza, peperoni, peperoncino e rucola. La combinazione mi stupisce e lui risponde, con un tono scherzoso: «Questo avevo oggi!» ${ }^{11}$. Nabaz, l'aiutocuoco, aggiunge: «La cucina è come il mare: non finisce mai!». Ne assaggio una forchettata: è buona, leggermente piccante. Sul bancone vengono disposte le teglie con la pasta, l'insalata mista, il pollo al forno, il pane e la frutta. Pierpaolo assegna a ogni volontario una portata oggi siamo in sei - e inizia la distribuzione del pranzo: per prime le donne, hanno la precedenza. Poi è il turno degli uomini. Tanti vogliono il sugo dell'arrosto e se ne fanno mettere anche sulla pasta. Quasi tutti chiedono più pasta e i volontari - con la pasta si può fare - aumentano la porzione. Ogni volontario ha il suo stile nel porgere il piatto e nell'accogliere le persone: alcuni di loro riconoscono gli utenti, li salutano per nome. La fila scorre. La maggior parte sono giovani dall'Africa subsahariana, arrivati da poco, ma ci sono anche uomini maturi e alcuni anziani. Qualcuno si muove con le stampelle e i volontari lo aiutano a portare il vassoio; alcuni passano davanti a noi indifferenti con gli auricolari sulle orecchie; alcuni sono in gruppo e si servono il pranzo parlando fra loro, altri rispondono al nostro saluto e al nostro sguardo. Alcuni hanno fretta, altri sono schivi e tengono lo sguardo basso, alcuni si irritano con gli altri in fila o con il volontario di turno. Ogni pomeriggio ci sono 200 persone di media: pranzano, si fanno una doccia calda, magari usufruiscono dell'ambulatorio medico, dei servizi legali o anche della residenza. Fino alle quattro e mezza la coda di persone è quasi ininterrotta. Nel corridoio c'è vita: dopo il pranzo le persone si salutano e scambiano quattro chiacchiere (Palutan, note di campo, 25 novembre 2016). 
Fondata nei primi anni Ottanta dai Gesuiti, la mensa del Centro Astalli è aperta dal lunedì al venerdì dalle 15.00 alle 16.30: fornisce circa duecento pasti ogni giorno ${ }^{12}$, quasi 59.000 pasti all'anno. Situata nelle cantine sotterranee dello storico quadrilatero della Compagnia di Gesù a Roma, in via degli Astalli, si snoda lungo un corridoio stretto sui cui lati si aprono piccole stanze: locali in cui mangiare, servizi igienici e docce, un ambulatorio medico, gli uffici in cui si fa orientamento legale e amministrativo. Sulle pareti dei locali sono appese grandi immagini che ritraggono gli ospiti del Centro Astalli intenti in varie attività: nella scuola di lingua italiana, durante una partita di scacchi, guardando una partita di calcio, intenti nel proprio lavoro, abbracciando un figlio. Sono quasi tutti primi piani, in cui risalta il volto delle persone e il loro sguardo. A commento di ogni foto, c'è un proverbio dei paesi di provenienza degli ospiti. Oltre a queste immagini, una sequenza di foto ricorda la visita di papa Francesco alla mensa nel settembre 2013 e il suo affacciarsi nelle salette dove i rifugiati stavano mangiando, il suo sostare con loro. Questa scelta iconografica mi viene spiegata da padre Camillo Ripamonti, presidente del Centro Astalli:

In primo piano c'è il viso delle persone, l'incontro tra sguardi. Perché è proprio questo il Centro Astalli: essere compagni di strada delle persone. E poi ci sono anche foto di persone che sono passate di qua ed è bello riconoscerle, rivederle in queste immagini, anche a distanza di tanto tempo (intervista a padre Ripamonti, 18 gennaio 2018).

I volontari e le volontarie svolgono un ruolo fondamentale nella mensa: cinquanta persone, la maggioranza delle quali sono donne, svolgono il proprio servizio una o più volte alla settimana in giorni prestabiliti. Il loro contributo non si limita alla distribuzione del cibo: sono infatti determinanti nel creare le condizioni migliori nell'accesso degli ospiti al pasto, facilitando il fluire della fila al bancone del self service, facendo attenzione che si rispettino i turni di arrivo, aiutando chi ha delle difficoltà a trasportare il vassoio, mediando fra le richieste di più cibo da parte degli ospiti e le richieste di una gestione attenta delle porzioni da parte del responsabile della cucina (le quantità di cibo fresco - carne, frutta e verdura - sono limitate e devono bastare per tutti, su richiesta si può abbondare invece con il primo piatto). Ogni volontario ha il proprio stile nel servizio e, dal momento che molti frequentano la mensa da quasi un decennio in giorni fissi, ci sono gruppi consolidati in cui gli stili personali si armonizzano fra loro. È raro dunque che la consegna del cibo sia un'azione asettica, anzi. È accompagnata da saluti di benvenuto agli ospiti, scambio con loro di commenti, domande, motti di spirito: brevissimi, veloci, a volte discreti, a volte plateali. È un susseguirsi di micro interazioni con potenzialità di comunicazione. Se un utente fa notare con irritazione che la carne del suo piatto è poco cotta, mostrarsi indifferenti da parte del volontario di turno o magari una sua risposta secca potrebbe allontanare o umiliare; ma quando il volontario, in un rumoroso italiano venato di romanesco, ammette annuendo: «il pollo è così poco cotto che è ancora vivo!» si dà spazio alla risata, che può essere contagiosa e propagarsi nella fila. Il comico che irrompe nella routine è «sfida ai sentimenti morali convenzionali» (Berger 2006: 13) e spezza la distanza fra donatori e beneficiari. Qui si palesa la questione sollevata da Harrell-Bond in un saggio del 1999, che noi non affrontiamo in questo lavoro, sull'asimmetria di potere tra strutture umanitarie e rifugiati. Certamente anche al Centro Astalli il tema dell'asimmetria che rinvia a modalità gestionali e relazionali è presente: gli utenti non possono scegliere cosa quando e quanto mangiare perché l'organizzazione quotidiana dei pasti per 250 persone, in questa come in altre strutture del genere, richiede una 
gestione complessa e attenta ${ }^{13}$. Quindi, il rischio di far sentire il beneficiario come dipendente dall'aiuto ricevuto e come soggetto non agente è reale. La figura dei volontari entra nella questione, ponendosi come un terzo soggetto che, proprio perché volontario, può interpretare il proprio ruolo con una libertà maggiore di quella che avrebbe un dipendente stipendiato. Può, per esempio, prendere bonariamente in giro il cuoco se la pasta si è scotta; può prendere le difese degli ospiti se questi si lamentano per qualche motivo; può dare qualche porzione più abbondante. Si tratta, come ben rileva il saggio di Larruina e Ghorashi riguardante un centro di richiedenti asilo in Olanda, di forme di micro-agentività, in cui l'introduzione di piccole, mirate, ma volute strategie di azione riescono a tener viva la motivazione dei volontari da un lato (Larruina, Ghorashi 2016) e a creare le premesse per una positiva negoziazione con il presente da parte dei rifugiati dall'altro.

31 Che senso danno i volontari al loro ruolo? Non possiamo fare osservazioni di ampio respiro, essendo la ricerca ancora in corso. Magda, volontaria in mensa dal 2009, se sollecitata con la domanda: c'è qualcosa a cui tieni, che cerchi di fare sempre quando vieni in mensa? risponde:

Io cerco di guardare le persone negli occhi, ma non sempre è possibile.

Magda spiega che quando ci sono molti utenti il movimento davanti al bancone è così veloce che le persone scorrono via senza nemmeno il tempo di salutarsi. Per questo, quando può, cerca di stare all'inizio o alla fine del self service:

Lì c'è il tempo, sono pochi secondi, per salutarsi, per dirsi due parole (intervista a

Magda, 8 dicembre 2017).

Il Centro Astalli non si limita alla mensa, ma si articola in un sistema più ampio che annualmente risponde alle necessità di circa 15.000 rifugiati e richiedenti asilo a Roma, mentre il numero raddoppia se si considerano le sedi in altre parti d'Italia ${ }^{14}$. È un mondo articolato che comprende strutture di accoglienza, servizi medici in convenzione con le ASL, accompagnamento legale, progetti di formazione, programmi di sensibilizzazione per la società civile, in particolare rivolti alle scuole. La mensa, tuttavia, è considerata il "cuore" di questo mondo:

È il cuore perché si è partiti da qui. Da un bisogno alimentare delle persone, fondamentalmente etiopi ed eritrei che stavano in Piazza Venezia ed erano in transito verso il Nord Europa e per i quali si era cominciato, negli anni ' 80 , a fare dei pacchi alimentari e a dare dei panini (intervista a padre Ripamonti, 18 gennaio 2018).

Padre Ripamonti racconta che il Centro Astalli ha iniziato le sue attività nel 1981, accogliendo l'appello dell'allora padre generale della Compagnia di Gesù, Pedro Arrupe, quando in Italia non esistevano ancora leggi sull'asilo. Nei 35 anni di attività la mensa ha mutato funzioni e struttura, pur rimanendo stabile la sede, adattandosi ai nuovi contesti legislativi e sociali: negli anni ' 90 , con le prime leggi sull'asilo, sono cambiate le provenienze e anche i progetti dei rifugiati, che diventavano più stanziali. Pertanto la mensa è divenuta un luogo in cui mangiare un pasto caldo e fermarsi a riposare. Per molti anni il Centro è rimasto il primo contatto dei rifugiati con Roma, il secondo approdo dopo lo sbarco in Sicilia, come afferma padre Ripamonti. Complice la crisi, sono aumentate le persone in situazioni di precarietà e di marginalità, faticando a mantenere un titolo di soggiorno valido. Per far fronte a queste esigenze, sono stati attivati servizi aggiuntivi. Il modello d'accoglienza di oggi e lo spirito che lo anima vengono spiegati da padre Ripamonti: 
Le persone vengono qui a mangiare, ma poi questo diventa uno spazio di interscambio, di interazione tra persone di provenienze affini quindi della stessa regione, della stessa etnia, della stessa nazione. È un luogo di aggregazione intorno al cibo. [...] Non c'è un modello precostituito, nel tempo si è cercato di capire quali fossero le modalità migliori di approccio, seguendo le tre parole che caratterizzano il Centro Astalli: accompagnare, servire, difendere (intervista a padre Ripamonti, 18 gennaio 2018).

\section{I due casi di studio a confronto}

Né il cuoco dell'Astalli, né le cuoche del Baobab considerano quello che cucinano cibo e basta, è sempre cibo addomesticato al gusto di chi è rivolto.

Pur essendo entrambe rivolte a rifugiati e richiedenti asilo, le due esperienze di accoglienza di cui ci siamo occupate hanno storia, stili, gerarchie indubbiamente diversi. Certamente però presentano anche dei denominatori comuni: per entrambe il contesto è Roma, da tempo luogo di passaggio di migranti dal Corno d'Africa che seguono percorsi collaudati nel tempo. Il legame con la città, sebbene rimosso dalla memoria istituzionale e flebilmente presente in quella locale, è tuttora presente nelle comunità di rifugiati somali e eritrei di più antico insediamento ${ }^{15}$ che hanno avviato attività commerciali e di ristorazione (erano queste che donavano le injere al centro Baobab). Entrambe le esperienze sono poi inserite in una rete ampia, che chiameremo del non-spreco, in cui soggetti sensibili - suddivisi in donatori di beni e donatori di tempo - concorrono tutti nell'assistere i bisognosi della capitale siano questi senza tetto, privi di mezzi, rifugiati o altro, orgogliosamente convinti che «a Roma non si muore di fame». Forni, panetterie, associazioni, sindacati, il Banco Alimentare, l'Elemosineria del Vaticano, la Caritas, varie parrocchie fanno parte di un circuito dove l'invenduto, l'avanzato, anziché ridursi in rifiuto, è rimesso in circolazione ${ }^{16} \mathrm{e}$, letteralmente, dà vita. Va poi detto che a questo tipo di solidarietà, che riconosce una comune umanità ${ }^{17}$, non fa riscontro una sensibilità istituzionale che a Roma, come in altre capitali europee, tende continuamente a spostare le persone in difficoltà ai margini spaziali e strutturali della città (vedi Agier 2018; Queirolo Palmas 2017). Sia il Baobab che l'Astalli devono far fronte ad azioni legate all'approvvigionamento del cibo, alla cottura e alla sua ricezione da parte degli utenti, il cui risultato non è scontato. Il gusto degli ospiti è sempre culturale, vale a dire interagisce con la dimensione memoriale, religiosa, relazionale (Lewis 2010). In entrambe le esperienze, la cottura degli alimenti cerca continuamente una mediazione tra il cibo disponibile e la modalità di preparazione perché venga accettato e consumato volentieri. Questa attenzione e questa tensione sono sempre presenti. Né il cuoco dell'Astalli, né le cuoche del Baobab, infatti, considerano quello che cucinano cibo e basta, è sempre cibo addomesticato al gusto di chi è rivolto. In entrambe le esperienze i cuochi dunque sono parte attiva e interpreti del contesto.

Benché le esperienze del Baobab e dell'Astalli abbiano il cibo come elemento centrale dell'accoglienza, appaiono portatori di modelli opposti: informale il Baobab, nato dal basso, costantemente minacciato nella sua esistenza da forze esterne quali polizia, istituzioni, condizioni ambientali sfavorevoli, destrutturato nelle sue forme e sospinto nelle periferie della città; infine, caratterizzato dall'informalità nei rapporti fra ospiti e volontari. In breve, un modello definibile come bottom up. L'altro, l'Astalli, riconosciuto dalle più alte cariche istituzionali laiche e religiose ${ }^{18}$ cuore di un sistema d'accoglienza articolato in tutta Italia, gestito da regole e da ruoli organizzati in una struttura 
gerarchica. In breve, un modello top down. Tuttavia, a ben guardare, il modello di bottom up del Baobab e quello top down dell'Astalli non si trovano su linee parallele destinate a non incontrarsi: Baobab Experience si è originata all'interno di un centro di accoglienza convenzionato con il Comune, che ha dovuto relazionarsi formalmente con le istituzioni, poi il contesto lo ha forzato a destrutturarsi. La mensa Astalli nasce come un'esigenza di volontariato che raccoglie le necessità di migranti in transito verso il Nord Europa; solo più tardi diviene più strutturata. In breve, entrambe le esperienze hanno saputo entrare in dialogo con le contingenze storiche, interpretare le necessità degli utenti e modificarsi nel tempo.

Sicuramente è diverso essere attivisti in un'esperienza di militanza - aggiungeremmo di tipo politico - piuttosto che essere operatori in un progetto più consolidato $\mathrm{e}$ riconosciuto; i primi impegnati in una tensione che mira a una orizzontalità dei compiti e a una fluidità dei ruoli, i secondi consapevoli di essere parte di un sistema verticale che prevede ruoli definiti. Se i volontari dei due casi di studio considerati appaiono collocarsi agli antipodi nelle modalità di gestione dell'accoglienza, esiste tutta una gamma di posizioni intermedie che vengono documentate e descritte in una recente e crescente letteratura sul tema - vedi in particolare i vari saggi usciti negli ultimi numeri di Antropologia Pubblica ${ }^{19}$. Per lo più si tratta di antropologi, ma anche psicologi, educatori, sociologi, che sono stati assunti come operatori in strutture quali gli SPRAR (Sistema Protezione Richiedenti Asilo e Rifugiati) o i CAS (Centri di Accoglienza Straordinaria) che si trovano ad affrontare situazioni in cui faticano a incidere sul sistema con le loro competenze trovandosi a operare in un eccesso di struttura. Nel caso del Baobab invece, in cui per inciso alcuni degli attivisti sono antropologi, il modello stesso d'accoglienza si basa sulla fluidità e sulla creatività dei suoi membri anche come risposta ai continui tentativi di scomposizione imposti da forze istituzionali (vedi il gran numero di sgomberi). Gli attivisti del Baobab si trovano dunque ad agire in uno stato continuo di precarietà che condividono con gli ospiti dei presidi, termine con il quale vengono abitualmente designati i campi. Tale aspetto di precarietà era già stato rilevato da Liisa Malkki che, una ventina d'anni fa, ha condotto ricerche in campi profughi in Tanzania e che ci ha lasciato sul tema pagine stupende (Malkki 1997). Malkki ci parla di un "campo fragile" che, in quanto tale, rende fragili gli abituali repertori interpretativi degli antropologi che si rilevano insufficienti a capire quello che avviene nel campo. Da qui la necessità di sperimentare linee di lavoro diverse. Partendo da questa sollecitazione, noi abbiamo adoperato la tecnica del photoeliciting con il fine di far affiorare un modello d'accoglienza che si veniva costruendo nel tempo per passare il testimone ad altri attivisti in una sorta di memoria dell'accoglienza. Le antropologhe hanno sollecitato gli attivisti del Baobab a far affiorare questa memoria considerandola un patrimonio che andava valorizzato e non disperso.

\section{Il senso del cibo in pratiche d'emergenza}

Il cibo è un oggetto complesso in cui le necessità materiali non sono scindibili da quelle simboliche della memoria o della commensalità.

I due casi etnografici che abbiamo presentato sono caratterizzati da due modelli di accoglienza diversi, ma comparabili in quanto in entrambi il cibo appare un'asse fondamentale attorno a cui si struttura l'accoglienza. Cambia nelle modalità (cosa viene cucinato, come e da chi) e cambia a seconda della contingenza. Nel caso del Baobab 
possiamo identificare tre momenti: la fase relativa al 2015, in cui il modello mantiene una certa continuità con l'esperienza del centro culturale (in cucina ci sono le cuoche eritree del ristorante coadiuvate dai volontari; la loro cucina si deve adattare alle necessità, per cui opta per la pasta, ma insaporita con spezie piccanti che richiamano il gusto africano; la fruizione dei pasti avviene all'interno del centro culturale). Nella fase del 2016 c'è una rottura netta con l'esperienza del centro Baobab a causa dello sgombero dei locali: manca la logistica (non c'è cucina, né magazzino in cui conservare il cibo); manca un luogo dedicato (si mangia nello spazio pubblico della strada); a cucinare non ci sono più cuoche ma i volontari a casa propria. Pertanto cambia la preparazione, la distribuzione e la fruizione del cibo. Tuttavia, rimane la consapevolezza che è cibo destinato a un gusto altro e pertanto si insaporisce con salse piccanti e spezie che richiamano la tradizione di chi viene da altrove. Infine, nella fase di piazzale Maslax, la gestione della situazione di emergenza diventa sempre più organizzata anche se le difficoltà di distribuzione si accentuano in quanto le auto con $\mathrm{i}$ pentoloni non possono arrivare fino il piazzale e il tendone. Rimane il fatto però che dietro a ogni piatto preparato si può riconoscere il volto di attivisti e volontari che hanno trasformato gli ingredienti in cibo che può essere più o meno buono, ma anonimo mai.

Nemmeno nella mensa del Centro Astalli il cibo è un fatto casuale, bensì frutto di scelte e di tentativi: dopo varie sperimentazioni, il cuoco ha optato per un menu con la principale qualità di «essere riconoscibile dagli utenti». È poi un cibo variato, che tiene presente le necessità nutrizionali e le direttive mediche (poco salato, ad esempio). È un cibo italiano - basato fondamentalmente sulla pasta - ma che non trascura i gusti dell'utenza, che lo preferisce speziato, né le sue preferenze e divieti alimentari. Sia al Baobab che all'Astalli si tratta di un cibo che intende creare relazione; infatti, nei piatti di ogni cuoco o cuoca è visibile il tentativo di avvicinarsi ai gusti dell'altro, in una volontà di mettersi in comunicazione. In entrambi i casi di studio il cibo rappresenta il cuore di un sistema di accoglienza che appare come un nurturing setting, un ambiente di cura in cui vengono offerti servizi miranti all'empowerment del singolo, in cui l'attenzione non è circoscritta all'aspetto nutritivo, ma abbraccia la persona nella sua interezza in cui le necessità materiali non sono scindibili da quelle simboliche della memoria o della commensalità.

Sia per il Baobab che per l'Astalli il cibo dipende dal dono di terzi e, in quanto tale, rimane un cibo d'emergenza. Tuttavia, prende le distanze da altri cibi sperimentati nell'assistenza a migranti forzati come ad esempio quelli pre-confezionati, distribuiti dalle agenzie umanitarie nei campi profughi i cosiddetti food-aid packages che Elizabeth Cullen Dunn, con una felice espressione, definisce «anti-food» (anticibo) la cui azione è «promuovere la passività, evidenziare l'assenza e esacerbare la perdita» (Cullen-Dunn 2011:140). Questo tipo di cibo, che viene distribuito nel campo georgiano di Tsimindatsqali sotto forma di pacchi di pasta precotta, chiamata macaroni, è descritto come «epitome of displacement» ossia simbolo dello sradicamento e «food from no place for people who had lost their places» perché, nella sua anonimità, «viene dal nulla e da nessuno». Riportando le parole di una abitante del campo si tratta «solo di calorie», «serve solo per mantenerti vivo, ma non c'è conforto in quel cibo» (Cullen Dunn 2011:142).

41 Sicuramente il cibo, nelle due esperienze da noi esaminate, dimostra di essere «un oggetto complesso incorporato in una rete di scambi e di interdipendenza» (Coleman 
2011) in cui il suo ruolo di nutrimento di socialità viene compreso e posto in atto. In questo senso richiama le forme di commensalità nell'accoglienza ai rifugiati a cui accenna brevemente Katarina Rozakou descrivendo il caso greco degli anni 2000 (Rozakou 2017). C'è dell'altro tuttavia: si tratta di un cibo che parla di solidarietà fra sconosciuti che si riconoscono in una condivisa umanità (Schmidt, Palutan, Gaborin 2017). Questo risulta molto chiaramente dalle interviste effettuate nel corso della nostra ricerca, in particolare agli attivisti del Baobab: «ogni essere umano deve aiutare l'altro nel modo che può» e «una parte del proprio tempo, qualcosina, tutti dovrebbero dedicarlo all'altro». Su questo tema, che richiama da vicino il concetto di comune umanità e di umanitarismo, avremo modo di soffermarci in altra opportunità ${ }^{20}$.

\section{Agentività come possibile chiave interpretativa}

Il cibo distribuito dagli attivisti del Baobab in via Cupa e dai volontari nella mensa del Centro Astalli cerca di contrastare la passività che sorge davanti all'aiuto ricevuto e alla routine dell'aiuto prestato attivando, dall'una e dall'altra parte, la capacità di rispondere come soggetti agenti nonostante le condizioni dettate dalle circostanze. Questo appare evidente quando gli attivisti del Baobab decidono di spostare all'interno delle loro case la cottura del cibo destinato ai rifugiati; quando si affidano a facebook per la gestione delle cuoche disponibili a prepararlo o ai volontari incaricati nell'andare a ritirarlo; quando si mobilitano per entrare nella rete del non spreco; quando gli ospiti del Baobab aderiscono a iniziative in ristoranti locali in cui sono loro i cuochi; quando si siedono attorno a fuochi del campo per compartire una bevanda calda o cucinare un piatto della tradizione che sarà spesso offerto anche ai volontari; quando da ospiti diventano loro stessi mediatori (anche se in questo saggio non abbiamo descritto questi due specifici punti). Per quanto riguarda il Centro Astalli, appare evidente quando il cuoco mette in atto tutti quegli accorgimenti perché il cibo da lui preparato venga accolto favorevolmente dagli ospiti; quando i volontari trovano il modo di interpretare il compito loro assegnato con maggiore libertà; quando gli ospiti colgono il senso dell'umorismo capace di attraversare il ponte comunicativo.

Su questa dimensione di agency vorremmo brevemente soffermarci per chiarire un concetto di cui spesso si parla, ma che altrettanto spesso rimane elusivo o confuso con termini affini quali intenzionalità, volontà, motivazione, creatività. Inoltre, essendo quasi sempre visto in interconnessione con il concetto di struttura, viene privato di una sua autonomia analitica che certamente non giova a una sua miglior comprensione. Le righe che seguono mireranno dunque a una sua scomposizione nei diversi elementi che lo costituiscono e a fare qualche osservazione sulla sua relazione con la struttura.

La prima variabile contro la quale considerare la dimensione di agentività è quella del contesto. Come sottolineano pragmatisti quali John Dewey e Herbert George Mead (Mead citato in Reck 1964), un'azione non può essere vista solo in termini utilitaristici che la situano nel conseguimento di un fine prestabilito ma astratto e dunque lontano dalla situazione concreta in cui viene a trovarsi l'attore sociale. Il fine non si ottiene senza tener conto del contesto specifico, il quale a sua volta è suscettibile di cambiamento e, pertanto, di una continua rivalutazione da parte del soggetto. Con loro, la distinzione kantiana tra interessi materiali e valori trascendenti è superata dal momento che ogni oggetto o fine è una costruzione sociale; posizionamento questo che pone le basi per una teoria dell'azione che analizza le condizioni di possibilità (Joas 
1993). In un altro saggio, Joas (1996) insiste nel vedere il carattere costitutivo dell'azione creativa completamente inserito in un contesto situazionale.

La seconda variabile, in costante divenire, è quella temporale. Le considerazioni iniziali partono da Mead (Joas 1985) che guarda agli attori sociali come immersi in momenti di passaggio con una continua revisione del loro posizionamento nei confronti del passato e del futuro in risposta a eventi emergenti nel presente. Elaborando da Mead, Emirbayer e Mische (1998) analizzano questa dimensione temporale tripartita, detta chordal triad, in cui l'agentività del soggetto si manifesta. La prima manifestazione è da loro chiamata iterativa in quanto si riferisce a modelli di pensiero e di azione originati nel passato e incorporati nella routine delle pratiche quotidiane, fornendo così stabilità e ordine tanto a identità soggettive quanto a realtà istituzionali. Questa dimensione iterativa si manifesta nella capacità del soggetto di richiamare alla memoria, selezionare e applicare schemi di azione ereditati dall'esperienza passata e applicarla in situazioni presenti o in proiezioni future. È questa una dimensione che richiama le teorie della pratica esemplificate da Bourdieu con il concetto di habitus (Bourdieu 1977) e da Anthony Giddens con il concetto di routine (Giddens 1984) guardando all'agentività come abituale, ripetitiva e scontata. La seconda dimensione, definita da Emirbayer e Mische proiettiva, si riferisce a possibili traiettorie di azione in cui gli attori sociali riconfigurano creativamente i modelli di pensiero e di azione ricevuti in vista di desideri e aspettative future. È un distanziarsi da abitudini e tradizioni per proiettarsi in una dimensione innovativa dove trovano posto piani e aspirazioni; è un costruire immagini che si proiettano nel futuro in una sfera dove gli attori sociali pensano di poter andare. La terza dimensione, pratica-valutativa, è la risposta alle contingenze del presente; essa richiede che la particolare situazione concreta sia riconosciuta come irrisolta e di conseguenza vengano cercate e valutate scelte possibili e infine decisa la direzione verso cui indirizzare l'azione.

46 Va chiarito che le tre dimensioni descritte non corrispondono a passato, presente futuro come stadi successivi di un'azione, ma piuttosto ciascuna di esse è simultaneamente orientata a passato, presente e futuro (Emirbayer, Mische 1998: 972). Questo è facilmente comprensibile se si considera che, nel cercare la risoluzione di una situazione concreta, si farà riferimento a uno schema abituale o iterativo richiamato dal passato e si darà una risposta nel presente tenendo in considerazione un più ampio progetto futuro. Tuttavia, se interpretiamo correttamente Emirbayer e Mische, una sola di queste tre dimensioni sarà predominante in ogni momento dato per cui un'azione sarà principalmente volta al passato, sollecitata dal presente o proiettata al futuro. Avere scomposto analiticamente le tre dimensioni temporali presenta il vantaggio di chiarire come gli attori sociali si muovano non soccombendo all'ambiente mutevole che li circonda.

47 La terza variabile da considerare è il carattere relazionale dell'agentività, un processo sempre dialogico attraverso il quale gli attori vengono a termini con gli altri (si veda, per esempio, Arendt 1977). È dunque la socialità dell'esperienza che stimola lo sviluppo delle capacità agenti.

Infine, l'agentività va vista in relazione con la struttura e con le modalità con cui si combina con le sue molteplici forme. Questo risulta del tutto evidente nella citata dimensione iterativa in cui le scelte poggiano su schemi e modelli preesistenti e profondamente assimilati che implicano strutture sociali di riferimento o comunque contribuiscono alla loro riproduzione. Sono proprio conoscenza e azione routinaria a 
rendere i sistemi organizzativi relativamente stabili e resistenti al cambiamento. Anche quando proiettata nel presente o nel futuro, l'agentività non si manifesta al di fuori della struttura bensì, come spiega Judith Butler, agisce dal suo interno entrando con essa in un dialogo serrato (Butler 1993). Per Giddens struttura e agentività sono elementi mutuamente costitutivi e, in quanto tali, inseparabili l'uno dall'altro (Giddens 1984). Questa tendenza a considerare i due elementi tanto intrecciati da risultare inseparabili è senza dubbio interessante; tuttavia porta anche a non poter analizzare separatamente le parti costitutive di ciascuno, negando loro un grado di autonomia. Invece, sostiene Archer (1988), questa autonomia deve essere preservata proprio perché diventa altrimenti impossibile esaminarne l'intreccio.

Tutta l'azione sociale è una sintesi da un lato plasmata e condizionata dal contesto temporale e relazionale dell'azione e dall'altro dall'agentività stessa che garantisce che l'azione sociale non sia mai completamente determinata o strutturata. A sua volta l'agentività non è mai completamente libera dalla struttura. Molteplici sono gli stimoli applicativi ai nostri specifici casi di studio, Baobab e Centro Astalli, che provengono da queste riflessioni analitiche sull'agentività dove i vari elementi considerati sono tutti riconoscibili: il contesto, la dimensione temporale, la variabile relazionale, gli attori sociali, la struttura.

Perché dunque in un momento di crisi e di emergenza scrivere sul cibo? In entrambi i casi presi in esame, il cibo nella sua valenza relazionale costituisce un elemento centrale di interazione e di costruzione comunicativa. Per il Baobab è il cibo, e le varie azioni a questo ricondotte, il canale privilegiato attraverso cui si è venuto progressivamente costruendo un modello d'accoglienza dal basso. Per il Centro Astalli il cibo e la sua accettazione sono al servizio della filosofia stessa su cui poggia il modello d'accoglienza proposto.

51 L'approccio generale adottato in questo saggio non ha posto l'accento sulle difficoltà sperimentate da attivisti, operatori e rifugiati quanto piuttosto sulle modalità creative nell'affrontarle. In quest'ottica, entrambi i casi di studio possono trovare nel concetto di agentività uno strumento capace di rendere visibili dinamiche che altrimenti rimarrebbero sottaciute.

\section{BIBLIOGRAFIA}

Agier M., 2018 La giungla di Calais. I migranti, la frontiera e il campo, Ombrecorte, Verona.

Ahearn L., 2001 «Language and Agency», in Annual Review of Anthropology, XXX: 109-137.

Allievi S., 2018 Immigrazione. Cambiare tutto, Laterza, Roma.

Altin R., Sanò G., 2017 «Richiedenti asilo e sapere antropologico. Una introduzione», in Rivista di Antropologia Pubblica, n. 3: 7-34.

Archer M., 1988 Culture and Agency: The Place of Culture in Social Theory, Cambridge University Press, Cambridge. 
Arendt H., 1977 The Life of the Mind, Harcourt, New York.

Bauman Z., 2016 Stranieri alle porte, Laterza, Bari.

Belloni M., 2016 «Refugees as Gamblers: Eritreans Seeking to Migrate through Italy», in Journal of Immigrant \& Refugee Studies, XIV n.1: 104-119.

Berger P.L., 2006 Homo ridens. La dimensione comica dell'esperienza umana, il Mulino, Bologna.

Biffi D., 2018 «Lavorare con richiedenti asilo e rifugiati: l'etnografia di un ricercatore-operatore», in Educazione Interculturale. Teorie, Ricerche, Pratiche, 16 (1): 1-21.

Borri G., 2017 «Humanitarian protraction status. The production of (im)mobile subjects between Turin and Berlin», in Etnografia e ricerca qualitativa, (1): 55-73.

Bourdieu P., 1977 Outline of a Theory of Practice, Cambridge University Press, Cambridge.

Brombin A., 2017 Coltivare il pianeta per coltivare se stessi. Viaggio tra gli ecovillaggi italiani, Franco Angeli, Milano.

Butler J., 1993 Bodies that Matter: On the Discursive Limits of "Sex", Routledge, New York.

Campesi G., 2011 «The Arab Spring and the Crisis of the European Border Regime: Manufacturing Emergency in the Lampedusa Crisis», in EUI Working Papers, RSCAS 59 Mediterranean Program.

- 2015 «Humanitarian confinement: an ethnography of reception centres for asylum seekers at Europe's southern border", in International Journal of Migration and Border Studies, 1(4): 398-418.

Centro Astalli, 2017, Rapporto Annuale 2017, Photopress, Roma.

Ciabarri L., 2016 «Introduzione», in L. Ciabarri (a cura di), I rifugiati e l’Europa. Tra crisi internazionali e corridoi d'accesso, Cortina, Milano.

Coleman L., 2011 «Introduction» in Coleman L. (ed.), Food: Ethnographic Encounters, Berg, OxfordNew York: 1-16.

Costantini O., 2012 «'Quando sono partito io'. Memoria individuale e memoria collettiva nei racconti di viaggio dei rifugiati eritrei», in Archivio Antropologico Mediterraneo no 14(2): 43-54.

Costantini O., Massa A., Yazdani J. (a cura di), 2016 Chi, cosa. Rifugiati Transnazionalismo e Frontiere, Mincione Edizioni, Roma.

- 2016 «Introduzione» in Costantini O., Massa A., Yazdani J. (a cura di), Chi, cosa. Rifugiati Transnazionalismo e Frontiere, Mincione Edizioni, Roma.

Counihan C., Siniscalchi V. (eds), 2014 Food Activism: Agency, Democracy and Economy, Bloomsbury Academic, London.

Counihan C., Van Esterik P. (eds), 2013 Food and Culture. A Reader, Routledge, New York-London.

Cramer J.M., Greene C.P., Walters L.M. (eds) 2011, Food as Communication/Communication as Food, Peter Lang, New York-Wien.

Cullen Dunn E., 2011 «The Food of Sorrow: Humanitarian Aid to Displaced People», in Coleman L. (ed.), Food: Ethnographic Encounters, Berg, Oxford-New York: 139 -149.

Cuttitta P., 2012 Lo spettacolo del confine. Lampedusa tra produzione e messa in scena della frontiera, Mimesis Edizioni, Milano.

Dewey J., 2014 [1925], Esperienza e Natura, Mursia, Milano. 
Dharod J. M., 2011 «Dietary Intake, Food Security, and Acculturation among Somali Refugees in the United States: Results of a Pilot Study», in Journal of Immigrant \& Refugee Studies, vol.9 (1): 82-97.

Dimitriadi A., 2017 «Governing irregular migration at the margins of Europe. The case of hotspots on the Greek islands», in Etnografia e ricerca qualitativa (1): 75-96.

Emirbayer M., Mische A., 1998 «What is agency?», in The American Journal of Sociology, CIII, 4: 962-1023.

Epstein I. et al., 2006 «Photo Elicitation Interview (PEI)», in International Journal on Qualitative Methods, $5(6): 1-9$.

Facchini D., 2018 «Alla deriva. I migranti, le rotte del Mar Mediterraneo, le ONG: il naufragio della politica che nega i diritti per fabbricare consenso» in Altreconomia, Milano.

Fassin D., 2007 «Humanitarianism as a Politics of Life», in Public Culture, IXX, n. 3: 499-520.

- 2012 Humanitarian Reason: A Moral Order of the Present, University of California Press, Berkeley.

Fassin D., Pandolfi M. (eds.), 2010 Contemporary States of Emergency: The Politics of Military and Humanitarian Intervention, Zone Books, New-York.

Federici S., Degli Esposti Merli E., 2014 Tutta la vita in un foglio, edizioni Lai-momo, Bologna.

Fekete L., 2018 «Migrants, borders and the criminalisation of solidarity in the EU», in Race \& Class, Institute of Race Relations, vol. 59(4):1-19.

Fiddian-Qasmiyeh E., Long K., Sigona N. (eds), 2014 The Oxford Handbook of Refugee and Forced Migration Studies, Oxford University Press.

Fisher M.F.K., 1989 [1943] The Gastronomical Me, North Point Press, San Francisco.

Fontanari E., 2017 «Afterword. An ethnographic gaze on power and refugees», in Etnografia e ricerca qualitativa, (1): 143-158.

Gallotti C., Tarabusi F., 2018 «Criticità e potenzialità della formazione e della comunicazione nei campi dell'accoglienza: un'introduzione» in Gallotti C. e Tarabusi F. (a cura di), Educazione Interculturale, numero monografico sul tema: Formazione e comunicazione nei campi dell'accoglienza di rifugiati e richiedenti asilo, vol.16(2): 1-8.

Gatta G., 2012 «Corpi di Frontiera. Etnografia del trattamento dei migranti al loro arrivo a Lampedusa», in Pizza G., Ravenda A. (a cura di), Presenze internazionali. Prospettive etnografiche sulla dimensione fisico-politica delle migrazioni in Italia, Numero monografico di AM rivista della Società italiana di Antropologia Medica, n.33\34: 129-162.

Gebrewold B., Bloom T., 2016 Understanding Migrant Decisions. From Sub-Saharan Africa to the Mediterranean Region, Routledge, Abingdon.

Giddens A., 1984 The Constitution of Society, University of California Press, Berkeley-Los Angeles. Giordan G., Zrinščak S., 2018 «One pope, two churches: Refugees, human rights and religion in Croatia and Italy», in Social Compass, vol. 65(1): 62-78.

Glasser I., 1988 More than Bread: Ethnography of a Soup Kitchen, University of Alabama Press, Tuscaloosa.

Gozzi G., Sorgoni B., 2010 I confini dei diritti. Antropologia, politiche locali e rifugiati, il Mulino, Bologna. 
Green S., 2013 «Borders and the relocation of Europe», in Annual Review of Anthropology, 42: 345-361.

Grønset A.S., 2013 «Being Human, Being Migrant. Senses of Self and Well-Being», in A.S. Grønset (ed.), Being Human, Being Migrant. Senses of Self and Well-Being, Berghahn Books, EASA Series, New York, XXIII no 1: 1-26.

Harrell-Bond B.E., 1999 «The Experience of Refugees as Recipients of Aid», in Ager A. (ed.) Refugees. Perspectives on the Experience of Forced Migration, Continuum, London: 136-68.

- 2005 Rights in Exile: Janus-Faced Humanitarianism, Berghahn, Oxford.

Harrell-Bond B.E., Voutira E., 1992 «Anthropology and the Study of Refugees», in Anthropology Today Vol. 8, No. 4: 6-10.

Harris N., Minniss R.F., Somerset S., 2014 «Refugees Connecting with a New Country through Community Food Gardening», in International Journal of Environmental Res Public Health, 11(9): 9202-9216.

Joas H., 1985 G.H. Mead: A Contemporary Re-Examination of His Thought, MIT Press, Cambridge, Mass.

- 1993 Pragmatism and Social Theory, University of Chicago Press, Chicago.

- 1996 The Creativity of Action, University of Chicago Press, Chicago.

Jourdan L., 2012 «Sono l'uomo giusto nel posto e nel momento sbagliato. Storia di un rifugiato eritreo a Kampala (Uganda)», in Antropologia 14: 257-273.

Kinya G. M., 2013, «Food aid intervention and gender: a case study of Kakuma refugee camp, Kenya», Institute of Anthropology, Gender \& African Studies (studies.uonbi.ac.ke)

Kiptinness C., Dharod J. M., 2011 «Bhutanese Refugees in the United States: their Dietary Habits and Food Shopping Practices upon Resettlement», in Journal of Hunger \& Environmental Nutrition, VI, 1: 75-85.

Korten D., 2015 Change the Story, Change the Future: A Living Economy for a Living Earth, BerrettKoehler Publishers, Pretoria.

Larruina R., Ghorashi H., 2016 «The Normality and Materiality of the Dominant Discourse: Voluntary Work Inside a Dutch Asylum Seeker Center», in Journal of Immigrant \& Refugee Studies, XIV n. 2: 220-237.

Lewis H., 2010 «Community Moments: Integration and Transnationalism at 'Refugee' Parties and Events», in Journal of Refugee Studies, XXIII n. 4: 571-588.

Malkki L., 1995a Purity and Exile: Violence, Memory, and National Cosmology among Hutu Refugees in Tanzania, Chicago University Press, Chicago.

- 1995b «Refugees and Exile: From "Refugee Studies" to the National Order of Things», in Annual Review of Anthropology, Vol. 24: 495-523.

- 1996 «Speechless Emissaries: Refugees, Humanitarianism, and Dehistoricization» in Cultural Anthropology 11(3): 377-404.

- 1997 «News and culture: Transitory Phenomena and the Fieldwork Tradition», in Gupta A., Ferguson J. (eds), Anthropological Locations. Boundaries and Grounds of a Field Science, University of California Press, Berkeley and Los Angeles: 86-101.

Manocchi M., 2012 Richiedenti asilo e rifugiati politici. Percorsi di ricostruzione identitaria, il caso torinese, Franco Angeli, Milano. 
- 2016 «Le sfide dell'accoglienza: passato e presente dei sistemi istituzionali di accoglienza per richiedenti asilo e rifugiati in Italia», in Meridiana. Rivista di storia e scienze sociali, 86: 121-143.

Manocchi M., Marchetti C., 2016 «Introduzione. Rifugiati in transito attraverso l'Europa», in Mondi Migranti: Rivista di studi e ricerche sulle migrazioni internazionali, 1: 21-38.

Marte L., 2008 «Migrant Seasonings: Food Practices, Cultural Memory, and Narratives of 'home' among Dominican Communities in New York City», PhD dissertation, The University of Texas at Austin.

Martinelli F., 2008 Periferia diffusa: i rifugiati di Stazione Tiburtina, Liguori, Napoli.

Mata Codesal D., 2008 «Rice \& Coriander. Sensorial re-creations of home through food: Ecuadorians in a northern Spanish city», Working Paper N. 50.

Meloni F., 2012 «'il mio futuro è scaduto’. Politiche e prassi di emergenza nelle vicende dei rifugiati in Italia», in Archivio Antropologico Mediterraneo, n. 14(1): 87-96.

Mintz S., 1996 Tasting food, tasting freedom, Beacon Press, Boston.

Morone A., 2011 «Dai respingimenti alla guerra civile. La Libia e I diritti dei migranti», in V. Maher (a cura di), Antropologia e diritti umani nel mondo contemporaneo, Rosenberg \& Sellier, Torino: 137-147.

Napuli L., 2015 «About the refugee movement in Kreuzberg/Berlin», in Journal für kritische Migrations-und Grenzregimeforschung 1 (2): 1-10.

Orlando G., 2015 «Food, social movements and solidarity. The view from anthropology», in Etnografia e ricerca qualitativa (2): 343-353.

Oteri C., Carrera F., 2012 Mediazioni Metropolitane. Rapporto di ricerca. Studio e sperimentazione di un modello di dialogo e intervento a favore dei richiedenti e titolari di protezione internazionale in situazione di marginalità, in www.centroastalli.it/wp-content/uploads/2014/02/mediazioni-metropolitanerapporto-di-ricerca.pdf.

Palumbo B., 2011 «Prefazione trans locale», in B. Sorgoni (a cura di), Etnografia dell'accoglienza. Rifugiati e richiedenti asilo a Ravenna, CISU, Roma: 7-13.

Paternò M.P. (a cura di), 2014 Questioni di confine. Riflessioni sulla convivenza giuridico-politica in una prospettiva multidisciplinare, Editoriale Scientifica, Napoli: 105-127.

Pinelli B. (a cura di), 2013a Annuario di Antropologia. Migrazioni e asilo politico n. 15, Ledizioni, Milano.

Pinelli B., 2013b «Fantasy, Subjectivity and Vulnerability through the Story of Woman Asylum Seeker in Italy», in A.G. Sigfrid (ed.), Being Human, Being Migrants. Sense of Self and Well-Being, Berghahn Books, New York-Oxford: 27-45.

Pinelli B., 2014 Campi di accoglienza per richiedenti asilo, in Riccio B. (a cura di), Antropologia e Migrazioni, CISU, Roma.

Pinelli B., 2017 «Borders, politics and subjects. Introductory notes on refugee research in Europe», in Etnografia e ricerca qualitativa, (1): 5-24.

Pinelli B., Ciabarri L. (a cura di), 2017 Dopo l'approdo. Un racconto per immagini e parole sui richiedenti asilo in Italia, editpress, Firenze.

Queirolo Palmas L., 2017 «Nuit debout. Transiti, connessioni e contestazioni negli accampamenti urbani dei rifugiati a Parigi», in Mondi Migranti, 2: 207-227. 
Rahola F., 2003 Zone definitivamente temporanee. I luoghi dell'umanità in eccesso, ombre corte, Verona.

Ravenda A., 2011 Ali fuori dalla legge. Migrazione, biopolitica e stato di eccezione in Italia, ombre corte, Verona.

Reck A. J. (ed.), 1964 George Herbert Mead: Selected Writings, Bobbs-Merrill, Indianapolis, IN.

Riccio B., Tarabusi F. (a cura di), 2018 Educazione Interculturale: Dilemmi, mediazioni e pratiche nel lavoro dell'accoglienza rivolta a rifugiati e richiedenti asilo, Numero monografico, vol. 16 n. 1.

Rozakou K., 2016 «Socialities of solidarity: revisiting the gift taboo in times of crisis», in Social Anthropology, XXIV, n. 2: 185-199.

Sbriccoli T., Jacoviello S. (eds), 2012 European perspectives on creolisation, Cambridge Scholars Publishing, Cambridge.

Sbriccoli T., Perugini N., 2012 «Dai Paesi di origine alle Corti Italiane. Campi, diritto e narrazioni nella costruzione della soggettività dei rifugiati» in Pizza G., Ravenda A. (a cura di), Presenze internazionali. Prospettive etnografiche sulla dimensione fisico-politica delle migrazioni in Italia, Numero monografico di AM rivista della Società italiana di Antropologia Medica, n.33\34-2012: 95-128.

Schiocchet L., 2018 «Anthropologists and refugees between global hegemony and the subaltern other» in Schiocchet L. (ed.), special issue Anthropologists and Refugees between the Middle East and Europe, The Journal Anthropology of the Contemporary Middle East and Central Eurasia (ACME) 4(2).

Schmidt D., Palutan G., Gaborin B., 2017 «Refugees, Food and Agency. Case-Studies from Italy» in V. Lajos, I. Povedák, T. Régi (eds) A találkozások antropológiája / The Anthropology of Encounters, MAKAT, Budapest: 195-205.

Schmidt D., Palutan G., 2018 «All'ombra del baobab. Rifugiati, emergenza e considerazioni sul dono alla periferia di Roma» in Dada Rivista di Antropologia post-globale, speciale n. 1, Debito e dono: 211-246.

Sciurba A., 2017 «Categorizing migrants by undermining the right to asylum. The implementation of the hotspot approach in Sicily» in Etnografia e Ricerca Qualitativa, 1/2017 (1): 97-120.

Segrè A., 2004 Lo Spreco Utile. Il libro del cibo solidale, Pendragon, Bologna.

Sorgoni B., Pozzi S., Gianfagna G., 2011 Etnografia dell'accoglienza. Rifugiati e richiedenti asilo a Ravenna, CISU, Roma.

Tarabusi F., 2014 «Politiche dell'accoglienza, pratiche della differenza. Servizi e migrazioni sotto la lente delle politiche pubbliche», in Archivio Antropologico Mediterraneo, 16 (1): 45-61.

Taran P. et al., 2016 Migranti e rifugiati hanno diritti. Rapporto Caritas Europa, Tao editrice, Todi.

Terzioglu A., 2018 «The banality of evil and the normalization of the discriminatory discourses against Syrians in Turkey» in Schiocchet L. (ed.), Special issue Anthropologists and Refugees between the Middle East and Europe, The Journal Anthropology of the Contemporary Middle East and Central Eurasia (ACME) 4(2).

Vacchiano F., 2005 «Cittadini sospesi: violenza e istituzioni nell'esperienza dei richiedenti asilo in Italia» in Annuario di Antropologia n.5 (2005): Rifugiati, a cura di M. Van Aken, n. 5: 1-17, Ledizioni, Milano.

Van Aken M. (a cura di), 2005 Annuario di Antropologia n.5 (2005): Rifugiati, Ledizioni, Milano. 
Van Gelder S. (ed.), 2014 Sustainable Happiness: Live Simply, Live Well, Make a Difference, BerrettKoehler Publishers, Pretoria.

Wall M. et al., 2017 «Syrian refugees and information precarity», in New media \& society, vol. 19(2): 240-254.

Webber F., 2017 «Europe's unknown war. Forced Migration and the Anthropological Response», in Race\&Class, vol. 59 (1): 36-53.

\section{ALLEGATO}

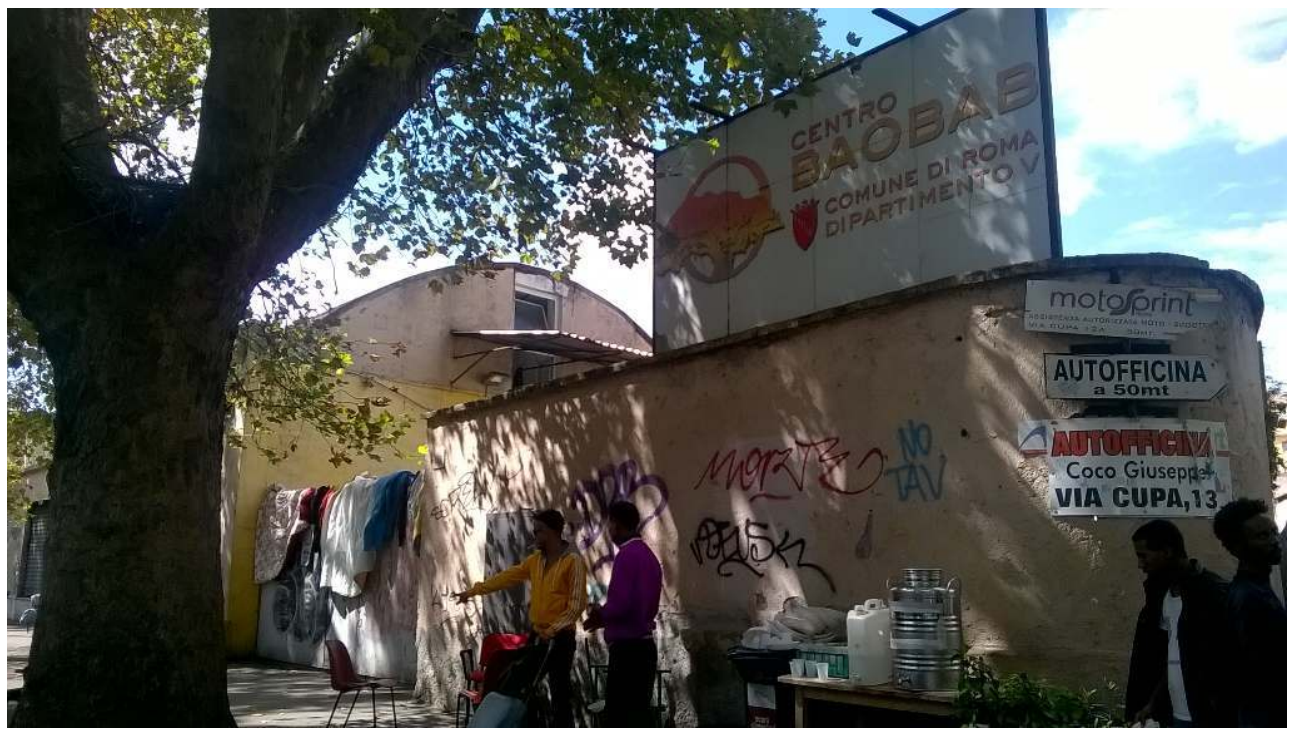

FOTO 1. «VIA CUPA È UNA TRAVERSA LUNGA E STRETTA SITUATA IN UN'AREA MANIFATTURIERA ABBANDONATA CHE TERMINA DI FRONTE ALLO STORICO CIMITERO DEL VERANO [...] IL CENTRO BAOBAB ERA UN'ASSOCIAZIONE GESTITA DA UN GRUPPO DI RIFUgIATI CHE LAVORAVA IN CONVENZIONE CON IL COMUNE» (๑ DONATELLA SCHMIDT). 


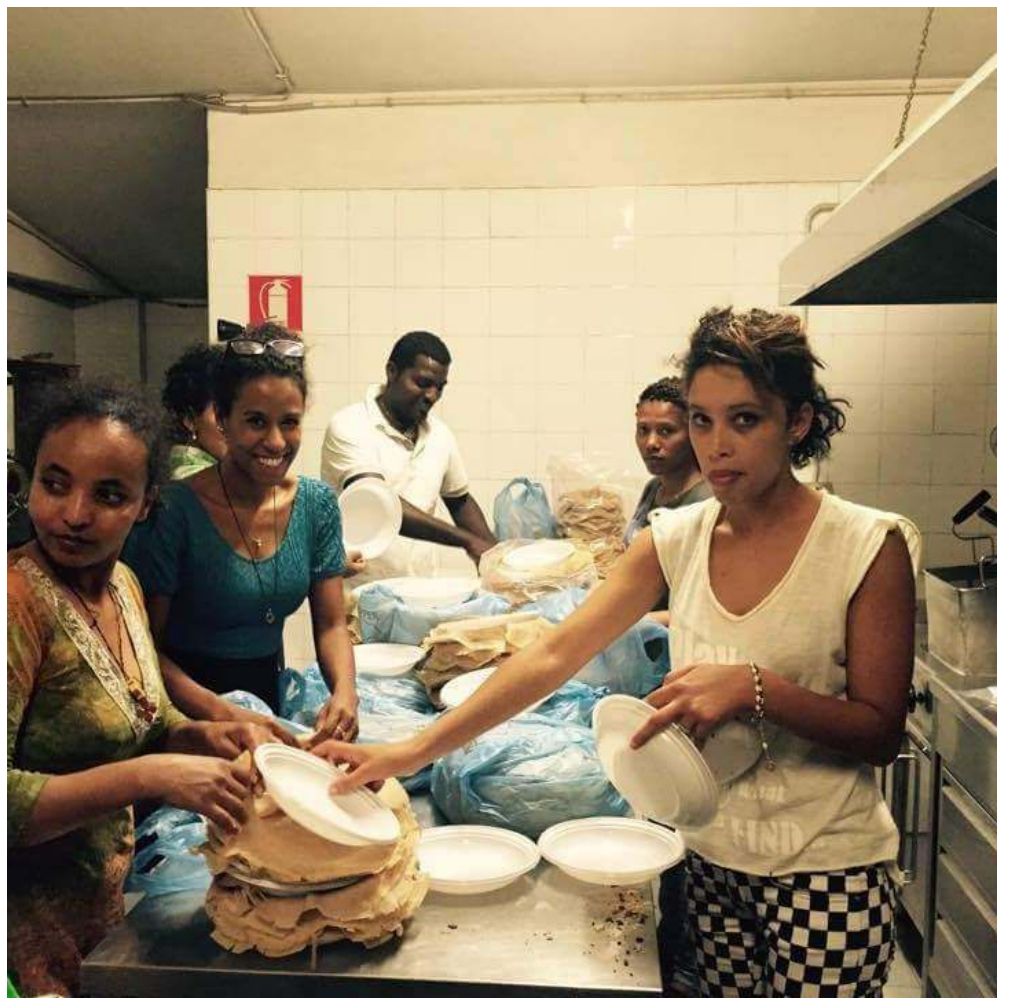

Foto 2. «Era la cucina il luogo nevralgico del Centro Baobab [...] La cucina era molto grande, c'erano due cuoche e, a turno, una ventina di volontari e di ospiti che aiutavano» (https://it-it.facebook.com/ BaobabExperience).

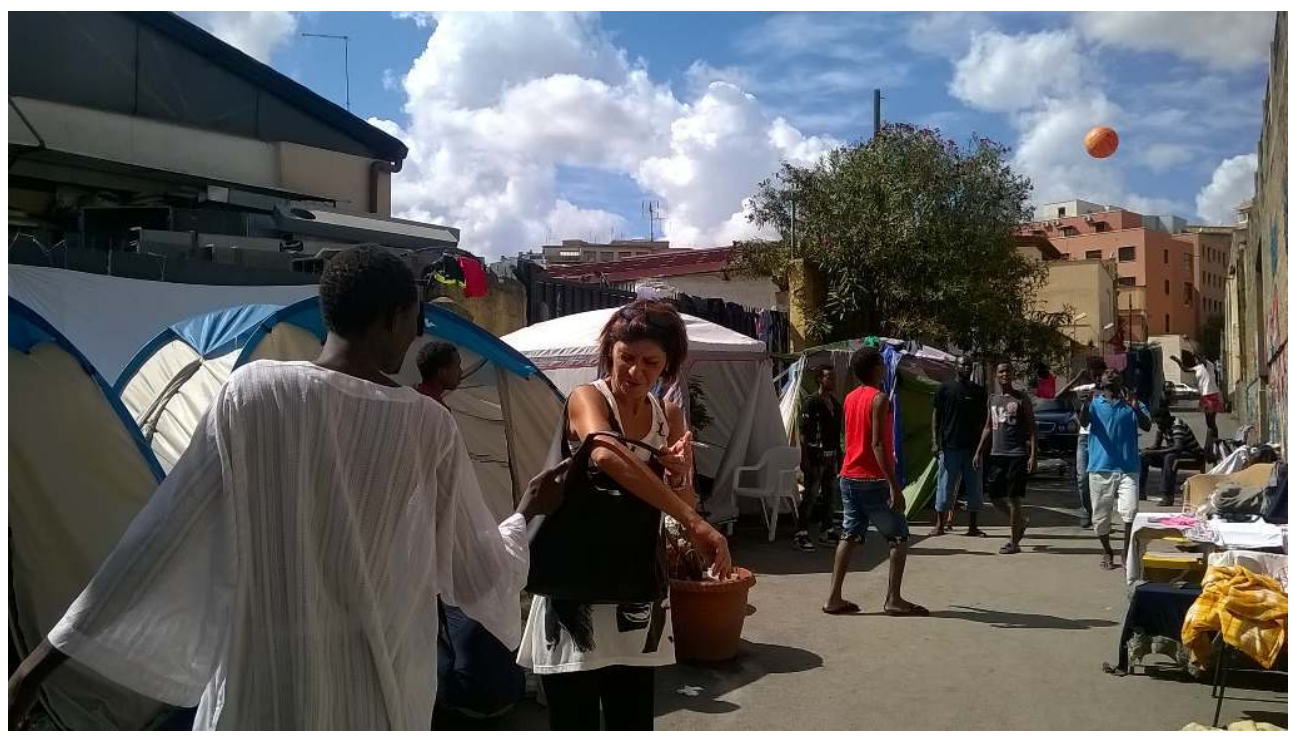

Foto 3. Con Baobab Experience «il Baobab riprende vita, ma non più dentro una struttura bensì sulla strada, dove si montano delle tende o si stendono materassi» (@ Donatella Schmidt). 


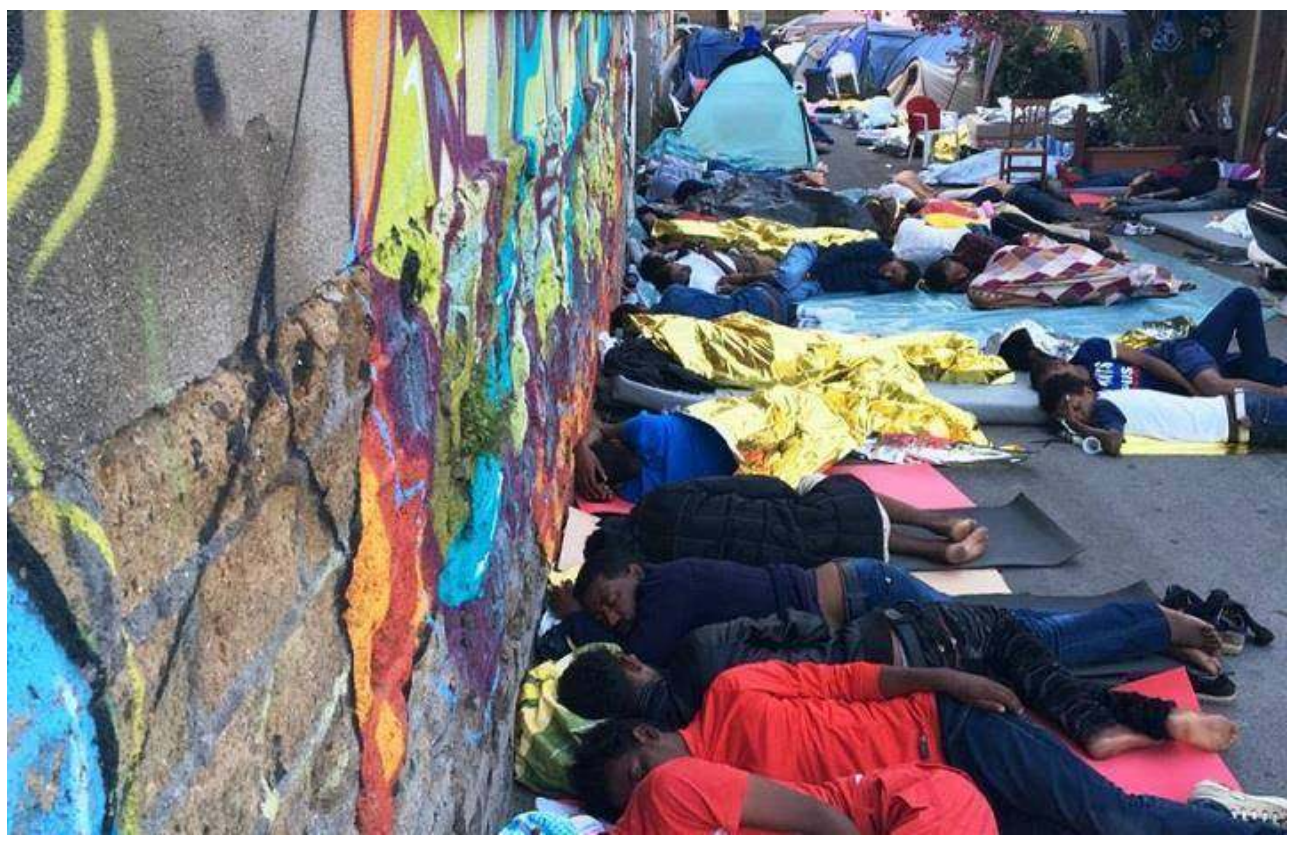

Foto 4. «La sera mettevamo delle sedie a chiudere la strada da una parte e dall'altra. Alle sette si smontava tutto, svegliavamo i ragazzi prima che aprissero i negozi e la strada veniva riaperta» (https://it-it.facebook.com/BaobabExperience).

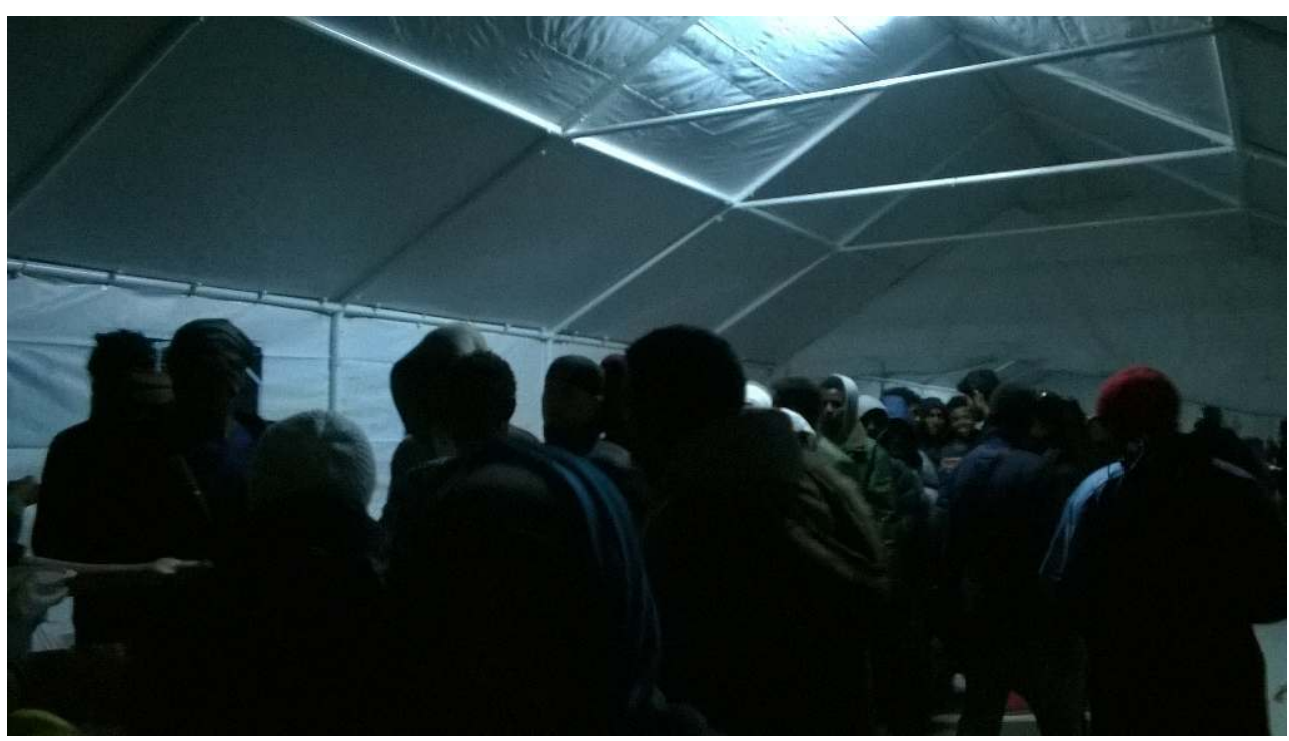

Foto 5. Piazzale Maslax: «il lungo tendone bianco funge da mensa e da ritrovo. All'avvicinarsi dell'ora di cena, le luci vengono accese e si riempie di giovani uomini e alcune poche donne fino a divenire affollato» (@ Donatella Schmidt). 


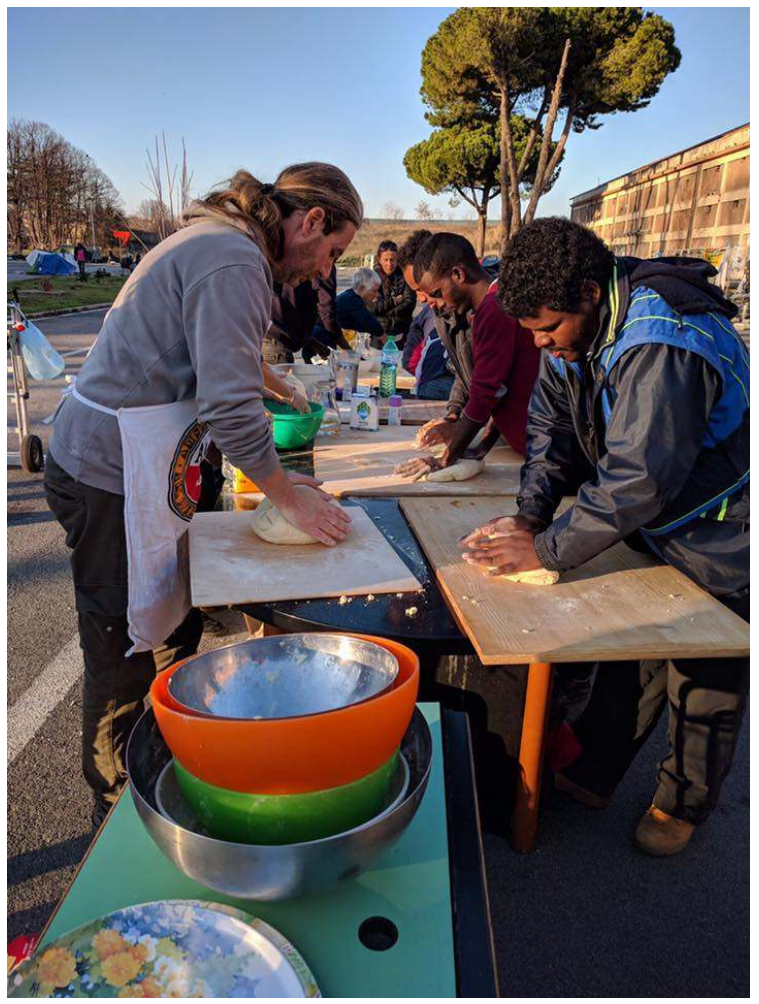

Foto 6. Piazzale Maslax, preparando gnocchi: «Anche in questa terza fase sperimentata dai volontari del Baobab il cibo continua a essere al centro dell'attenzione e della relazione» (๑ Donatella Schmidt)

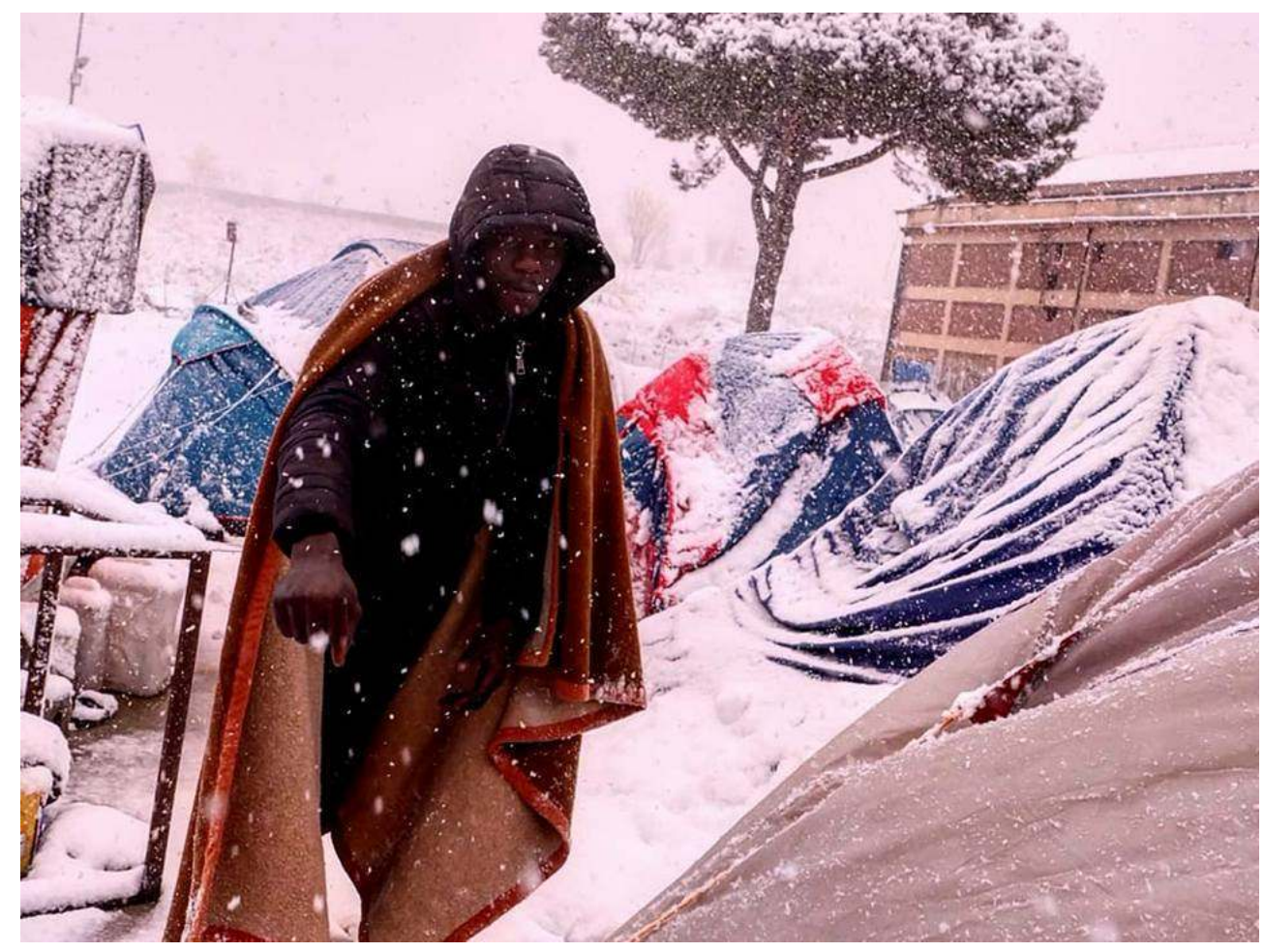

Foto 7. Piazzale Maslax, nevicata di febbraio: «Quello che offriamo noi è una tenda, un pranzo, la cena e amicizia, ma a livello di benessere, di bagni, di una doccia, di un letto, di una camera al coperto non c'è» (@ Donatella Schmidt). 


\section{NOTE FINALI}

1. I dati etnografici presentati fanno parte di una ricerca tutt'ora in corso, condotta all'interno dell'unità di ricerca che fa capo al progetto FOR - Food and Refugees. La ricerca guarda principalmente al ruolo del cibo in luoghi di emergenza o di precarietà all'interno di grandi città europee (per es. Roma, Berlino) che vedono la presenza di rifugiati nelle varie accezioni del termine. Parti di questa ricerca sono state presentate in due convegni internazionali a Göttingen (Germania) e a Debrecen (Ungheria); Göttingen è stata l'occasione per entrare in contatto con una rete di studiosi che affrontano tematiche simili e per partecipare alla stesura collegiale di un progetto europeo; il convegno di Debrecen era centrato sull'incontro in ambito antropologico ed è risultato in una miscellanea in cui le autrici hanno pubblicato un saggio con materiale raccolto a Bassano e a Roma (Schmidt, Palutan, Gaborin 2017). Questo saggio è stato composto a quattro mani. Tuttavia, Donatella Schmidt è responsabile per le parti 1, 2.2, 3.1, 3.3, 5, 7; Giovanna Palutan per le parti 2.1, 3.2, 4, 6.

2. Va menzionato anche l'importante compendio The Oxford Handbook of Refugee and Forced Migration Studies che affronta il tema da angolazioni diverse.

3. Il Centro Baobab era nato da un'esperienza di occupazione di un capannone abbandonato di proprietà delle Ferrovie dello Stato situato dietro la stazione Tiburtina, che negli anni Novanta era stato occupato da rifugiati e da richiedenti asilo provenienti dal Corno d'Africa e che il Comune aveva chiuso per questioni igienico-sanitarie. Era noto come Hotel Africa.

4. L'injera è il caratteristico pane spugnoso della cucina eritrea.

5. "Francesco, don Pietro e i volontari dell'associazione Baobab", di Gianguido Vecchi, Corriere della Sera, 1 aprile 2018 (https://roma.corriere.it/notizie/cronaca/18_aprile_01/francesco-donpietroe-baobab-41f9766c-34ff-11e8-8de8-ad207e8187ca_print.html); "Via Cupa, leggere Szymborska ai migranti del Baobab”, di Mariagrazia Calandrone, Corriere della Sera, 3 agosto 2016 (https://roma.corriere.it/notizie/cronaca/16_agosto_02/via-cupa-leggere-szymborska-migrantibaobab-3d836fc2-58e5-11e6-b011-ed7749260a21.shtml\#); Il centro Baobab a Roma ’̀ un antidoto contro il razzismo, di Nicola Lagioia, Internazionale, 27 luglio 2016 (http://intern.az/1upL).

6. Va detto che gli attivisti, già a partire dalla chiusura del Centro Baobab, avevano chiesto alle istituzioni una sede idonea ad accogliere i transitanti anche in previsione degli arrivi l'estate successiva. Questa richiesta alle istituzioni è avvenuta attraverso tre modalità: la partecipazione a tavoli istituzionali; l'attività di sensibilizzazione dell'opinione pubblica attraverso i media con l'invito a fotografi e giornalisti al campo di via Cupa e attraverso l'organizzazione di eventi culturali e di manifestazioni (marce, cortei, sit in); infine, l'occupazione di uno stabile abbandonato di proprietà della Regione, risultante in un immediato sgombero da parte delle forze dell'ordine.

7. Sulle dinamiche e sulle motivazioni decisionali della migrazione eritrea in transito attraverso l'Italia vedi, per esempio, l'articolo di Milena Belloni. La Belloni rileva come gli eritrei si sentano non stabili e di come questa percezione impedisca loro di apprendere la lingua e di cercare lavoro. La percezione deriva dal fatto di considerare il viaggio compiuto non in linea con le loro aspirazioni e con quelle delle famiglie rimaste a casa. In Eritrea il raggiungimento dell'età adulta coincide con l'abilità di provvedere agli anziani e ai minori, condizione frustrata da un servizio militare obbligatorio, prolungato e poco pagato. Se il paese di immigrazione presenta condizioni economiche tali da allungare i tempi delle rimesse a casa o da non permetterle affatto, l'esperienza migratoria si considera fallita (Belloni 2016). Sul tema vedi il lavoro di Gebrewold e Bloom, Understanding Migrant Decisions. From Sub-Saharan Africa to the Mediterranean Region (2016), e il testo Chi, cosa curato da Costantini, Massa, Yazdani (2016).

8. Spartitraffico collocati dalle forze dell'ordine per disincentivare la creazione di altri campi spontanei.

9. L'intervista è stata condotta da entrambe le ricercatrici nel maggio del 2015. 
10. Per una visione dell'utenza così come per una descrizione della sua struttura operativa, rinviamo al Rapporto Annuale pubblicato dal Centro Astalli: http://centroastalli.it/rapportoannuale/

11. La cucina della mensa dipende sia dal budget a disposizione, con cui si comprano la carne e i prodotti freschi, sia dalle scorte e dal cibo donato, che può arrivare anche all'ultimo momento.

12. L'afflusso diminuisce durante il digiuno del Ramadan e nei mesi estivi quando molti si spostano nelle zone turistiche e agricole per lavori stagionali.

13. È del tutto evidente che regolamentare la preparazione dei pasti o l'ingresso alla mensa risponde a logiche di gestione che hanno un impatto sulla sfera del sé dei richiedenti asilo e sulla loro capacità di azione e, come conseguenza, contribuiscono a sottolineare una situazione di tipo asimmetrico. Sul tema si veda, per esempio, il saggio di Biffi che rileva come lo scontro tra le esigenze di gestione di un centro d'accoglienza da parte degli operatori e le esigenze di vivere il centro come una casa da parte dei rifugiati entrino in contrasto, divenendo spesso causa di incomprensioni da parte di entrambi (Biffi 2018).

14. Palermo, Catania, Trento, Vicenza, Napoli, Milano, Padova.

15. Si consideri, per esempio, il Quartiere africano in cui targhe o monumenti in rovina celebravano le conquiste italiane o una tutta una serie di scrittori e registi somali ed eritrei di seconda generazione - Igiaba Scego, Ribka Sibhatu, Cristina Ali Farah, per citarne solo alcuni che forzano a riflettere sul passato coloniale italiano e la sua eredità.

16. Sulla questione del non spreco legato a un più ampio sistema di responsabilità sociale $\mathrm{e}$ ambientale citiamo i lavori di David Korten (2015), Sarah Van Gelder (2014) e Alice Brombin (2017). Andrea Segrè, fondatore del Last Minute Market si è occupato delle dimensioni economiche, sociali e ambientali dello spreco, (ricordando che sono 8,4 miliardi sprecati in un anno solo in Italia, ossia circa 7 euro settimanali a famiglia), elaborando un circuito di recupero che permette di ridistribuire il cibo in scadenza (2004). Varie sono le iniziative nazionali e le pratiche a livello locale su questo tema: vanno menzionati il progetto Family Bag, il progetto Reduce (Ricerca, Educazione e Comunicazione: un approccio integrato per la prevenzione degli sprechi alimentari: http://www.sprecozero.it/cose-il-progetto-reduce/), i protocolli antispreco firmati dal Comune di Milano e di Cesena, il progetto "Roma non spreca" (https://www.comune.roma.it/pcr/it/ newsview.page?contentId=NEW148300) per il recupero delle eccedenze alimentari da destinare a progetti di solidarietà sociale, reso possibile dalla legge n. 155 del 25 giugno 2003, detta del "Buon Samaritano", che facilita la distribuzione degli alimenti non più commercializzabili da parte delle organizzazioni di utilità sociale senza scopo di lucro.

17. Su questo tema vedi Rosakou 2017; Schmidt, Palutan 2018.

18. La mensa, che ha una convenzione con il Comune, è stata visitata da Papa Francesco, gesuita anche lui, e da varie figure istituzionali tra le quali il Presidente della Repubblica Sergio Mattarella.

19. Campesi 2015; Gallotti, Tarabusi 2018; Biffi 2018, per citarne alcuni.

20. Per una discussione sul concetto di umanitarismo - fondato su una profonda convinzione dell'uguale dignità dell'essere umano in quanto tale e del conseguente obbligo di assicurare a chiunque il minimo indispensabile alla vita - cfr., per esempio, Harrell-Bond (1999), Fassin (2007) e Grønset (2013).

\section{RIASSUNTI}

Il saggio verte sulle pratiche legate alla cottura e alla distribuzione del cibo in due esperienze d'accoglienza rivolte a rifugiati e a richiedenti asilo nella periferia di Roma. Il primo caso riguarda un modello di ospitalità dal basso conosciuto come Baobab; il secondo riguarda un'esperienza gestita dai gesuiti, nota come mensa del Centro Astalli. Due principali domande 
guideranno il nostro lavoro: qual è il senso del cibo per attivisti e volontari nei due esempi considerati? In che modo pratiche legate al cibo sono in grado di creare spazi di comunicazione e di riflessività tra rifugiati e settori della società ospitante? L'approccio generale adottato in questo saggio non pone l'accento tanto sulle difficoltà sperimentate da attivisti, volontari e rifugiati, che sono una costante della loro interazione quotidiana, quanto sulle modalità creative nell'affrontarle. In quest'ottica, si suggerisce che entrambi i casi di studio potrebbero trovare nel concetto di agentività uno strumento capace di rendere visibili dinamiche che altrimenti rimarrebbero sottaciute.

This essay aims to tie food practices to refugees and asylum seekers who live in temporary or emergency settings, focusing on two specific ethnographic cases located in the city of Rome. The first case refers to a bottom up hospitality experience named Baobab; the second case refers to a more structured experience, a soup-kitchen devoted to asylum seekers run by the Jesuit International Service for Refugees. Two main questions will lead our work: what is the sense of food for activists and volunteers in the two case-studies under consideration? And, how are food practices capable of creating spaces of communication and reflexivity among refugees and sectors of the host society? The general approach adopted in the essay is not so much on difficulties experienced by volunteers and refugees alike, which are present in everyday interaction, but rather on creative ways to cope with them. In this perspective, we suggest that both case-studies might find in the concept of agency a tool capable of making visible dynamics that would otherwise remain unspoken.

\section{INDICE}

Keywords : food, refugees, Rome, emergency, shelters, agency

Parole chiave : cibo, rifugiati, Roma, emergenza, agentività

\section{AUTORI}

\section{DONATELLA SCHMIDT}

Università degli Studi di Padova

donatella.schmidt@unipd.it

GIOVANNA PALUTAN

Università degli Studi di Padova

giovanna.palutan@gmail.com 